\title{
Appraising Kirchhoff approximation theory for the scattering of elastic shear waves by randomly rough defects
}

\author{
Stewart G Haslinger ${ }^{\mathrm{a}, *}$, Michael J S Lowe ${ }^{\mathrm{a}}$, Peter Huthwaite ${ }^{\mathrm{a}}$, Richard V Craster ${ }^{\mathrm{b}}$, Fan Shi $^{\mathrm{c}}$ \\ ${ }^{a}$ Department of Mechanical Engineering, Imperial College, London \\ ${ }^{b}$ Department of Mathematics, Imperial College, London \\ ${ }^{c}$ Department of Mechanical and Aerospace Engineering, Hong Kong University of Science and Technology
}

\begin{abstract}
Rapid and accurate methods, based on the Kirchhoff approximation (KA), are developed to evaluate the scattering of shear waves by rough defects and quantify the accuracy of this approximation. Defect roughness has a strong effect on the reflection of ultrasound, and every rough defect has a different surface, so standard methods of assessing the sensitivity of inspection based on smooth defects are necessarily limited. Accurately resolving rough cracks in non-destructive evaluation (NDE) inspections often requires shear waves since they have higher sensitivity to surface roughness than longitudinal waves. KA models are attractive, since they are rapid to deploy, however they are an approximation and it is important to determine the range of validity for the scattering of ultrasonic shear waves; this range is found here. Good agreement between KA and high fidelity finite element simulations is obtained for a range of incident/scattering angles, and the limits of validity for KA are found to be much stricter than for longitudinal wave incidence; as the correlation length of rough surfaces is reduced to the order of the incident shear wavelength, a combination of multiple scattering and surface wave mode conversion leads to KA predictions diverging from those of the true diffuse scattered fields.
\end{abstract}

Keywords: Diffuse scattered field, elastic shear waves, randomly rough surface, Kirchhoff approximation, Non-destructive evaluation

\section{Introduction}

The roughness of a surface is well known to significantly alter the scattering of elastic waves in solids, and therefore has an important impact on applications such as NDE ultrasonic inspection in the nuclear industry [1, seismic wave exploration at infrasonic frequencies 2, 3, and boundary phonon-scattering to model thermal transport in nano-structures [4]. All of these research problems are governed by theoretically similar wave scattering models, albeit with different scales of wavelength. The content of this article is generally applicable, but the illustrative examples focus on application to NDE inspection and the frequency and wavelengths are chosen accordingly.

The reflection of ultrasound from cracks is affected by the surface characteristics of the insonified region: with increasing roughness, the specularly reflected signals are reduced, with the energy being spread over a diffuse range of angles. However, roughness is not only an intrinsic property of the surface; the parameters of the incoming waves also influence the measurement of the scattered response. The "effective" roughness [1] of a surface is increased for smaller incident wavelength and when the incident wave direction coincides with the local surface normal. Therefore any analytical model must incorporate both surface and wave parameters, inevitably making the elastic extensions of acoustic models, to include polarisation and mode conversion, considerably more challenging.

\footnotetext{
${ }^{*}$ Corresponding author

Email address: s.haslinger@imperial.ac.uk (Stewart G Haslinger)
} 
Historically, the most popular approaches to study acoustic wave scattering have applied perturbation theory, reviewed for example by [1, 5] and the Kirchhoff approximation (KA) 6, 7]. As mentioned by [8], it is well known that perturbation techniques are reliable for surfaces with low roughness, whereas KA works well for the larger range of roughness that is pertinent to the fields of NDE and seismology. The fundamental concept of KA is that a scatterer may be discretised as a finite number of facets, each of which is assumed to be part of an infinite tangential plane insonified by the incident wave.

Specific geometries of defects are very rarely known in practical NDE applications so it is desirable to predict an expected scattered field based on surface statistics, which are attainable. In this way, a proposed industrial inspection can be assigned an expected sensitivity threshold.

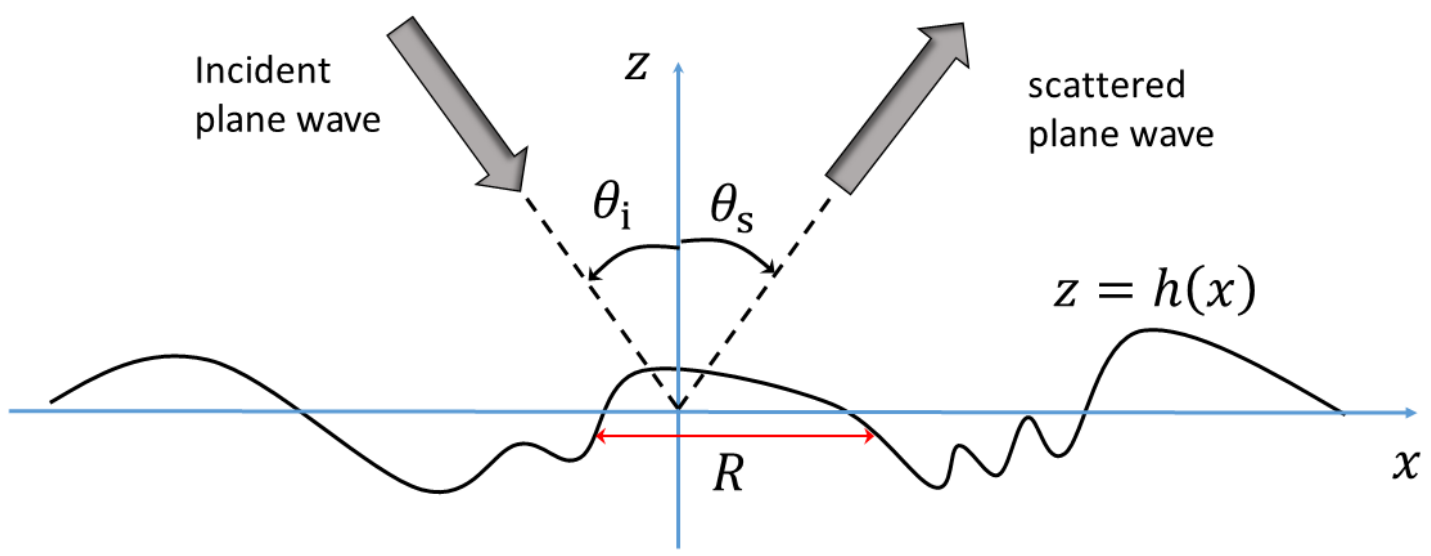

Figure 1: A plane wave scattered by a rough surface in 2D with global incident and scattering angles $\theta_{\mathrm{i}}, \theta_{\mathrm{s}}$. The height data of the surface is defined by $z=h(x)$, and $R$ is the separation between any two points of the surface.

A scattered ultrasonic wave is often described as the sum of two parts: the coherent field and the diffuse field. The direction of the coherent field is defined by the specular angle $\left(\theta_{\mathrm{s}}\right.$ in Figure 1) and the phase is constant, relative to that of the incident wave, for all surfaces of the same statistical class of roughness. The diffuse field inherits the random nature of the rough surface, with both its direction and phase varying over a wide range of angles.

In the case of KA analysis of acoustic scattering, theoretical formulae for both the coherent field [6] and the diffuse field 1, 9, using statistical roughness parameters, have long been known; the equivalent coherent field expression for elastodynamic scattering incorporates a straightforward adjustment of the wavenumber. However, the diffuse term, derived using a slope approximation obtained from integration by parts, proved to be far more elusive with the main difficulty arising from mode coupling at the rough boundary [1].

The recent breakthrough by [8] used a stationary phase analysis of the full KA expressions for the scattered field to derive expected value formulae for longitudinal incidence for both 2D and 3D surfaces, within the valid region of KA [10]. These analytical solutions may be used without the necessity for multiple simulations, since they are not surface-specific, depending only on statistical roughness parameters, wavenumbers and angles of incidence and scattering. The case of incident shear waves is not a trivial extension of these methods, and the first step, undertaken here, is to establish the range of validity for KA for the scattering of elastic shear waves.

KA validation using numerical techniques has mostly been performed in $2 \mathrm{D}$ for better computational efficiency, but is also justfied by the fact that quasi-2D cracks (where one of the dimensions is much greater than the others) are common in practice, for example within welds where cracks have been found to follow weld beads [1]. In addition, the phenomena that determine the limits of validity for KA are equivalent in $2 \mathrm{D}$ and $3 \mathrm{D}[12$ and, therefore, restriction to 2D-scattering allows for a greater range of crack dimensions 
and characteristics to be investigated [13. The models of [10] utilised a state-of-the-art finite element (FE) method incorporating the GPU-driven software package Pogo [14 that greatly accelerates elastodynamic simulations.

Most studies of elastic wave scattering by rough surfaces have illustrated and tested models with longitudinal wave incidence. For instance, although 1, 10, 15 have all derived and/or mentioned shear wave incidence, no illustrative examples or KA validity ranges were provided. Indeed, a definitive range of validity for KA for the case of incident SV elastic waves appears to be missing from the literature. The shorter wavelength of shear waves naturally indicates greater sensitivity to roughness, but a quantification of the range of validity of $\mathrm{KA}$ for shear wave incidence is becoming increasingly important. Owing to their heightened sensitivity, shear waves are widely used in NDE inspections to detect rough cracks. It is clear that the diffuse part of the field will become dominant as roughness increases and here we show how this transition arises more rapidly for shear waves in comparison with longitudinal incidence, and consequently affects the valid region of shear wave KA.

The article is organised as follows: Section 2 introduces the statistical profile and generation method for the $2 \mathrm{D}$ rough surfaces. Section 3 summarises the derivation of the KA method and expressions. Section 4 describes FE simulations with two alternative types of SV-wave excitation, one being a pure plane wave, and the other being a tapered plane wave with a Gaussian profile. Results are provided in Section 5, including a range of validity for KA for shear wave incidence, followed by a detailed discussion of multiple scattering and surface wave mode conversion in Section 6. Concluding remarks are drawn together in Section 7.

\section{Randomly rough surfaces in $2 \mathrm{D}$}

The 2D geometry for a rough defect is illustrated in Figure 1, where the global incident and scattering angles are denoted by $\theta_{\mathrm{i}}$ and $\theta_{\mathrm{s}}$, respectively. The accompanying unit wavevectors are defined as

$$
\boldsymbol{k}^{\mathrm{inc}}=\left(\sin \theta_{\mathrm{i}},-\cos \theta_{\mathrm{i}}\right) ; \quad \boldsymbol{k}^{\mathrm{sc}}=\left(\sin \theta_{\mathrm{s}}, \cos \theta_{\mathrm{s}}\right) .
$$

A rough surface is usually described in terms of its deviation from a smooth reference surface, with the emphasis on two factors: the spread of heights about the mean surface and the lateral variation of these heights along the surface.

We define the function $h(x)$ to represent the height of the surface relative to $z=0$, where $x$ denotes the 1D position on this reference surface. The surface heights are described by a statistical height distribution with zero mean:

$$
<h>=\int_{-\infty}^{\infty} h p(h) \mathrm{d} h=0,
$$

where $p(h) \mathrm{d} h$ is the probability of a surface point being at a height between $h$ and $h+\mathrm{d} h$ away from the mean surface. The probability density function $p(h)$ for a Gaussian surface is

$$
p(h)=\frac{1}{\sigma \sqrt{2 \pi}} \exp \left(-\frac{h^{2}}{2 \sigma^{2}}\right),
$$

where $\sigma$ is the RMS height defined (with an ergodic assumption [5]) as

$$
\sigma=\sqrt{<h^{2}>}=\sqrt{\frac{1}{N} \sum_{i=1}^{N} h_{i}^{2}} .
$$

This standard deviation gives us the height scale of the rough surface; the lateral variation of $h$ is expressed using a correlation function, which is also assumed to be Gaussian:

$$
C(R)=\frac{<h(x) h(x+R)>}{\sigma^{2}}=\exp \left(-\frac{R^{2}}{\lambda_{0}^{2}}\right) .
$$


The second statistical parameter, $\lambda_{0}$, is called the correlation length, and is the distance over which the correlation function $C(R)$ falls by $1 / e$. The variable $R$ is the distance between any two points on the surface, as shown in Figure 1.

In choosing Gaussian distributions for the height and correlation functions, we follow preceding literature 8, 10, 1, 7, 15, 16. As well as the analytical benefits of using a well-understood statistical distribution, experimental evidence has indicated that rough surfaces possess Gaussian spectra when arising from natural processes, such as thermal fatigue [15, 17. However, recent work [18] advocates an autoregressive method to accurately represent real rough surfaces in 3D and recommends caution when assuming Gaussian roughness, advising that it performs well under certain conditions, but not universally.

The weighted moving average process, developed by Ogilvy [16, is used to generate multiple realisations of randomly rough surfaces that depend on the statistical parameters $\sigma$ and $\lambda_{0}$, which are prescribed as multiples of the incident shear wavelength $\lambda_{\mathrm{s}}$. The method is described in detail in [16 but basically involves convolving a sufficiently large series of uncorrelated random numbers with a smaller set of weighting functions, that are defined using the desired surface discretisation and its correlation length $\lambda_{0}$. In this way, the uncorrelated data points are smoothed to represent a rough surface with a predetermined RMS height $\sigma$.

It is crucial to the stochastic method that although each realisation is different (due to the randomness), it may be classified by $\sigma, \lambda_{0}$. Thus, a sufficiently large number of realisations, say $N=200$, produces statistically meaningful results to determine the validity of KA for each pair of $\sigma, \lambda_{0}$. Another important detail is that the rough surfaces themselves are statistically meaningful. Ogilvy [16] provided a guideline that a sample of length $50 \lambda_{0}$ is more than adequate for the sample statistics to be representative. We consider correlation lengths varying from half a wavelength to two wavelengths for incident shear waves with a wavelength of around $0.8 \mathrm{~mm}$ for a centre frequency of $4 \mathrm{MHz}$ in aluminium. An initial dataset of length $50 \mathrm{~mm}$ is suitably large to provide statistical stability for aluminium samples, but is of no practical interest for industrial applications. Therefore, we extract samples of smaller length from the initial $50 \mathrm{~mm}$ datasets, to obtain examples of interest that are statistically representative.

\section{Elastodynamic Kirchhoff approximation theory for SV-wave incidence}

As explained in the Introduction, section 1. Kirchhoff approximation (KA) theory has been extensively and successfully used to model acoustic and elastodynamic scattering from rough surfaces. Derivations are widely accessible so we omit much of the detail provided by, amongst others, 11, 19, 20, choosing instead to focus on the differences that shear, rather than longitudinal, wave incidence brings. Since we are analysing the $2 \mathrm{D}$ problem, we consider vertically shear (SV) waves in the classical sense [19. Suffice to say, as with much of the preceding literature [8, 10, 1, 21, we apply the stress-free boundary condition.

\subsection{Kirchhoff approximation}

KA is illustrated in Figure 2(a) for SV wave incidence. The motion of a single surface point is assumed to be the same as if it were part of an infinite tangential plane. The total displacement at this point is approximated as a summation of the incident SV-wave and the reflected shear-shear (S-S) and shearlongitudinal (S-P) waves:

$$
\boldsymbol{u}^{\mathrm{KA}}=A^{\mathrm{sv}}\left(\boldsymbol{d}_{0}+r_{\mathrm{sS}} \boldsymbol{d}_{\mathrm{s}}+r_{\mathrm{sp}} \boldsymbol{d}_{\mathrm{p}}\right)
$$

where the vectors $\boldsymbol{d}_{0}, \boldsymbol{d}_{\mathrm{s}}, \boldsymbol{d}_{\mathrm{p}}$ are the displacement polarisation vectors and $r_{\mathrm{ss}}, r_{\mathrm{sp}}$ are reflection coefficients of $\mathrm{S}$ and $\mathrm{P}$ waves respectively. SV-wave incidence means that the shear wave polarisation vector is in-plane, and perpendicular to the directional vector, as indicated by the arrows in Figure 2(a).

Comparing expression (6) with the longitudinal case (see equation (8) in [10]), there is an additional factor $A^{\mathrm{sv}}$ :

$$
A^{\mathrm{sv}}=-\frac{1}{\cos \alpha_{\mathrm{s} 0}} \hat{\boldsymbol{d}}_{0} \cdot \hat{\boldsymbol{t}}
$$

where $\hat{\boldsymbol{d}}_{0}$ is the unit polarisation vector for the global incident field and $\hat{\boldsymbol{t}}$ is the local unit tangent vector, defined at each point on the surface, as illustrated in Figure 2(b). 


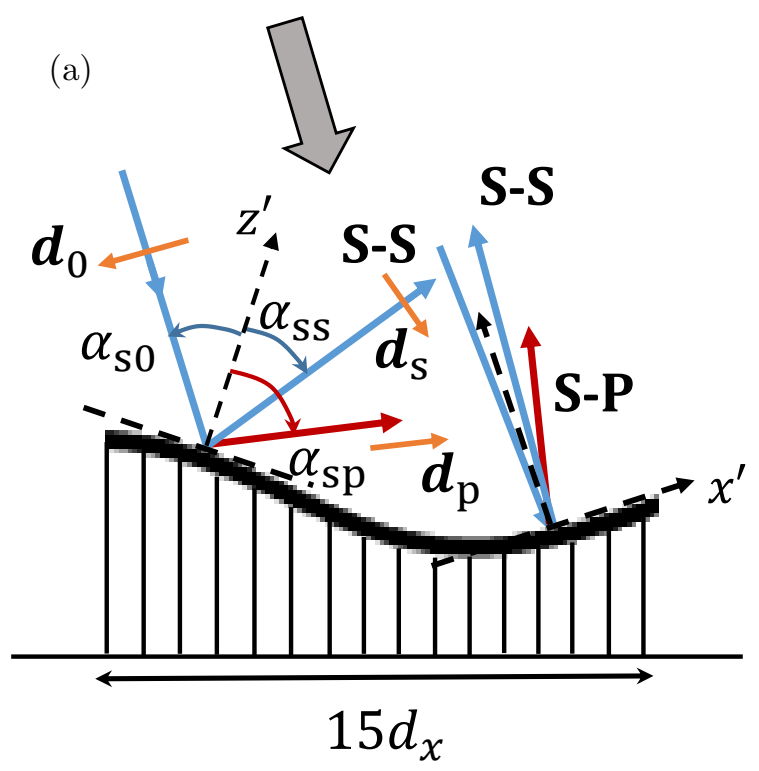

(b)

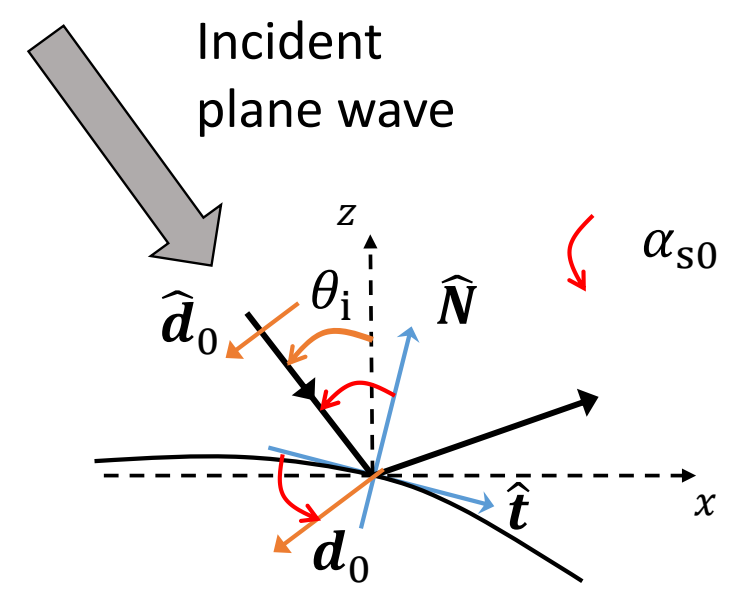

Figure 2: Illustration of KA discretization and local parameters for rough surface scattering of an incident plane SV-wave. (a) Shear-shear (S-S) and shear-longitudinal (S-P) wave directions and polarisation vectors are shown, as well as local coordinates $x^{\prime}, z^{\prime}$ for each facet of length $d_{x}$ and local incident and reflected S-S and S-P angles $\alpha_{\mathrm{s} 0}, \alpha_{\mathrm{ss}}, \alpha_{\mathrm{sp}}$. (b) Local tangent $\hat{\boldsymbol{t}}$, local normal $\hat{\boldsymbol{N}}$ and local and global polarisation vectors, $\boldsymbol{d}_{0}$ and $\hat{\boldsymbol{d}}_{0}$ respectively, for incident shear waves are shown.

The direction of the local polarisation vector $\boldsymbol{d}_{0}$ varies by 0 or $\pi$ from that of the global polarisation vector $\hat{\boldsymbol{d}}_{0}$, which is fixed, according to the local curvature of the surface. The factor (7) accounts for this change of sign, being a normalised dot product that guarantees

$$
A^{\mathrm{sv}} \boldsymbol{d}_{0}=\hat{\boldsymbol{d}}_{0} .
$$

The amplitude (7) is required to maintain the sign consistency for all the waves, hence its role in the expression for the total displacement (6).

\subsection{Reflection coefficients}

The local reflection coefficients $r_{\mathrm{ss}}, r_{\mathrm{sp}}$ are derived following [19], assuming a traction-free boundary condition on the boundary $z^{\prime}=0$ of a local coordinate system for each facet, and therefore, each tangential plane as in Figure 2(a). Thus, the coefficients depend on the local scattering angles and the wavenumbers $k_{\mathrm{s}}, k_{\mathrm{p}}$ :

$$
r_{\mathrm{ss}}=\frac{\sin 2 \alpha_{\mathrm{s} 0} \sin 2 \alpha_{\mathrm{sp}}-\kappa^{2} \cos ^{2} 2 \alpha_{\mathrm{s} 0}}{\sin 2 \alpha_{\mathrm{s} 0} \sin 2 \alpha_{\mathrm{sp}}+\kappa^{2} \cos ^{2} 2 \alpha_{\mathrm{s} 0}} ; r_{\mathrm{sp}}=\frac{-\kappa \sin 4 \alpha_{\mathrm{s} 0}}{\sin 2 \alpha_{\mathrm{s} 0} \sin 2 \alpha_{\mathrm{sp}}+\kappa^{2} \cos ^{2} 2 \alpha_{\mathrm{s} 0}},
$$

where $\kappa$ is the ratio of shear to longitudinal wavenumbers.

There is a crucial difference between the incident shear and incident longitudinal cases that underpins much of the contrast in the scattering behaviour. The mode-converted reflected angle, $\alpha_{\mathrm{sp}}$, can become complex since it is defined by

$$
\frac{\sin \alpha_{\mathrm{s} 0}}{\sin \alpha_{\mathrm{sp}}}=\frac{1}{\kappa} ; \quad \kappa=\frac{k_{\mathrm{s}}}{k_{\mathrm{p}}}>1 .
$$

Thus, there is a critical incident angle $\alpha_{\mathrm{s} 0}=\alpha_{\text {crit }}$ defined by

$$
\alpha_{\mathrm{crit}}=\sin ^{-1}\left(\frac{1}{\kappa}\right) .
$$


The analogous mode-converted P-S case for longitudinal incidence shares a similar condition to (10), but with the reciprocal ratio of sines, ensuring that $\alpha_{\mathrm{ps}}$ is always real. Note that in 10 , we define a condition on $\kappa$ which is true for most media, and all that are relevant for ultrasonic NDE. As an example, aluminium typically has $\kappa \simeq 2$, which determines a critical incident angle of $\alpha_{\text {crit }} \simeq 30^{\circ}$ for shear wave incidence.

In the event of $\alpha_{\mathrm{s} 0}>\alpha_{\text {crit }}$, the reflected S-P wave propagates parallel to the surface with exponentially decaying amplitude relative to the depth of the material, i.e. surface wave mode-conversion. The mode conversion is complicated further by the roughness of the surface whose undulations result in additional multiple scattering of the surface wave. As mentioned in the Introduction, section 1 neither of these factors are accounted for by KA, although a correction to the contribution to the total displacement at each facet of the discretisation is implemented here. Facets where surface wave mode conversion occurs are identified within our KA code, i.e. when the local angle

$$
\alpha_{\mathrm{s} 0}>\alpha_{\mathrm{crit}} \Longrightarrow \alpha_{\mathrm{sp}}>\pi / 2
$$

and an adjustment to the local reflection coefficients $r_{\mathrm{ss}}, r_{\mathrm{sp}}$ is made [19], p.179:

$$
\begin{aligned}
& r_{\mathrm{ss}}=-\exp (-2 i \xi) ; \\
& r_{\mathrm{sp}}=\frac{\exp \left(-i \xi+\tau k_{\mathrm{s}}\right) \sin 4 \alpha_{\mathrm{s} 0}}{\sqrt{\kappa^{2} \cos ^{4}\left(2 \alpha_{\mathrm{s} 0}\right)+4\left(\kappa^{2} \sin ^{2}\left(\alpha_{\mathrm{s} 0}\right)-1\right) \sin ^{2}\left(2 \alpha_{\mathrm{s} 0}\right) \sin ^{2}\left(\alpha_{\mathrm{s} 0}\right)}},
\end{aligned}
$$

where the parameters $\xi, \tau$ are introduced for complex $\alpha_{\mathrm{sp}}$, with the polarization vector $\boldsymbol{d}_{\mathrm{p}}$ in $(6)$ changed accordingly, with $\gamma=\arg \left(\alpha_{\mathrm{sp}}\right)$ :

$$
\begin{aligned}
\tan \xi & =\frac{2 \sqrt{\left(\kappa^{2} \sin ^{2} \alpha_{\mathrm{s} 0}-1\right)} \sin 2 \alpha_{\mathrm{s} 0} \sin \alpha_{\mathrm{s} 0}}{\kappa \cos ^{2} 2 \alpha_{\mathrm{s} 0}}, \\
\tau & =\frac{\sinh \gamma}{\sqrt{\sinh ^{2} \gamma+\cosh ^{2} \gamma}}
\end{aligned}
$$

\subsection{Formulation of scattering problem}

The total displacement field is given by

$$
\boldsymbol{u}=\boldsymbol{u}^{\mathrm{sc}}+\boldsymbol{u}^{\mathrm{inc}}
$$

with the scattered field represented by the elastodynamic Helmholtz integral formula given by Ogilvy [1] p.136, and Achenbach et al. [20, as well as recent publications [10, 8]:

$$
u_{k}^{\mathrm{sc}}(\boldsymbol{R})=\int_{\mathcal{S}(\boldsymbol{r})} \Sigma_{i j k}(|\boldsymbol{R}-\boldsymbol{r}|) u_{i}(\boldsymbol{r}) n_{j}(\boldsymbol{r}) d \mathcal{S}(\boldsymbol{r}),
$$

where $\Sigma_{i j k}$ is Green's stress tensor, $\boldsymbol{R}$ is the location of the observation point, $\boldsymbol{r}$ is a point on the rough surface $\mathcal{S}(\boldsymbol{r}), \boldsymbol{n}$ is the outward unit normal vector to the surface and $u_{i}(\boldsymbol{r})$ is the $i$ th component of the KA boundary displacement (6). Note that since we are considering $2 \mathrm{D}$, the $k=2$ component of scattered displacement $u_{k}^{\mathrm{sc}}$ is zero and the dummy indices $i, j \neq 2$ with the index values 1 and 3 corresponding to the $x$ and $z$ directions respectively (see Figure 1 ).

Using far-field assumptions 21] $R \gg r$ and $k_{\alpha} R \gg 1$, where the index $\alpha$ denotes the incident wave-type i.e. shear wave in this case, (14) may be simplified as

$$
\boldsymbol{u}^{\mathrm{sc}}(\boldsymbol{R})=-i k_{\beta} \sqrt{\frac{2 \pi i}{k_{\beta}}} \int_{\mathcal{S}_{0}} \frac{e^{i k_{\beta} D}}{4 \pi \sqrt{D}} \boldsymbol{U}_{\mathrm{s} \beta}(\boldsymbol{D}, \kappa) d \mathcal{S}_{0} .
$$


Here, we have converted the integration over the rough surface to one over the mean line $\mathcal{S}_{0}($ i.e. $z=0$ ) by using

$$
\boldsymbol{n} d \mathcal{S} \approx \boldsymbol{N}_{0} d \mathcal{S}_{0}, \quad \boldsymbol{N}_{0}=-\boldsymbol{i} \frac{\partial h}{\partial x}+\boldsymbol{k}
$$

where $\boldsymbol{i}$ and $\boldsymbol{k}$ are the unit orthonormal basis vectors. In effect, the integration may be performed over small rectangular elements of the rough surface, parallel to the local tangent [1] p.79.

Note also the use of the vector $\boldsymbol{D}=\boldsymbol{R}-\boldsymbol{r}$ in $[15$, with associated magnitude $D$ and unit vector $\hat{\boldsymbol{D}}$. Since $R$ represents the far-field, further approximations may be used, as with the stationary phase approach of [8], but here we are primarily interested in determining the range of validity of the KA theory and proceed to integrate 15 numerically. The KA appears in the complicated boundary displacement term $\boldsymbol{U}_{\mathrm{s} \beta}$ where the index $\beta$ denotes the scattered wave-type, which can be shear s or longitudinal, $\mathrm{p}$ :

$$
\begin{aligned}
& \boldsymbol{U}_{\mathrm{sp}}(\hat{\boldsymbol{D}}, \kappa)=\left[\left(\boldsymbol{u}^{\mathrm{KA}} \cdot \boldsymbol{N}_{0}\right)\left(1-\frac{2}{\kappa^{2}}\right)+\frac{2}{\kappa^{2}}\left(\boldsymbol{u}^{\mathrm{KA}} \cdot \hat{\boldsymbol{D}}\right)\left(\boldsymbol{N}_{0} \cdot \hat{\boldsymbol{D}}\right)\right] \hat{\boldsymbol{D}} \\
& \boldsymbol{U}_{\mathrm{ss}}(\hat{\boldsymbol{D}}, \kappa)=\left(\boldsymbol{N}_{0} \cdot \hat{\boldsymbol{D}}\right) \boldsymbol{u}^{\mathrm{KA}}+\left(\boldsymbol{u}^{\mathrm{KA}} \cdot \hat{\boldsymbol{D}}\right) \boldsymbol{N}_{0}-2\left(\boldsymbol{u}^{\mathrm{KA}} \cdot \hat{\boldsymbol{D}}\right)\left(\boldsymbol{N}_{0} \cdot \hat{\boldsymbol{D}}\right) \hat{\boldsymbol{D}} .
\end{aligned}
$$

The functions $\boldsymbol{U}_{\mathrm{s} \beta}(\hat{\boldsymbol{D}}, \kappa)$ depend on the local incident and scattered angles and the roughness of the surface in the terms $\boldsymbol{u}^{\mathrm{KA}}$ in equation $(6), \boldsymbol{N}_{0}$ in equation $(16)$ and $\hat{\boldsymbol{D}}$, as well as the physical parameters in the wavenumber ratio $\kappa$. The standard bulk wave equations relating wavenumber to wave-speed are expressed as

$$
k_{\mathrm{s}}=\frac{\omega}{c_{\mathrm{s}}}=\omega \sqrt{\frac{2 \rho(1+\nu)}{E}} ; \quad k_{\mathrm{p}}=\frac{\omega}{c_{\mathrm{p}}}=\omega \sqrt{\frac{\rho(1+\nu)(1-2 \nu)}{E(1-\nu)}} .
$$

It follows that equation 15 is actually a sum of two integrals:

$$
u^{\mathrm{sc}}(\boldsymbol{R})=\sqrt{\frac{2 \pi k_{\mathrm{p}}}{i}} \int_{\mathcal{S}_{0}} \frac{e^{i k_{\mathrm{p}} D}}{4 \pi \sqrt{D}} \boldsymbol{U}_{\mathrm{sp}} d \mathcal{S}_{0}+\sqrt{\frac{2 \pi k_{\mathrm{s}}}{i}} \int_{\mathcal{S}_{0}} \frac{e^{i k_{\mathrm{s}} D}}{4 \pi \sqrt{D}} \boldsymbol{U}_{\mathrm{ss}} d \mathcal{S}_{0} .
$$

Numerical integration of 20 is carried out for specific realisations of rough surfaces and compared with the scattered fields obtained from FE models for plane wave excitation. The rough surfaces are discretised into small rectangular facets of length $d_{x}=\lambda_{s} / 35$, and the orientation of each facet is given by the surface slope at its midpoint. Equation $(20)$ is then written as the summation of contributions from $N$ facets:

$$
\begin{aligned}
u^{\mathrm{sc}}(\boldsymbol{R}) & =\sqrt{\frac{2 \pi k_{\mathrm{p}}}{i}} \sum_{n=1}^{N} A_{n} \int_{\mathcal{S}_{n}} \frac{e^{i k_{\mathrm{p}} D_{n}} e^{i k_{\mathrm{s}} \boldsymbol{r} \cdot \boldsymbol{k}^{\mathrm{inc}}}}{4 \pi \sqrt{D_{n}}} \boldsymbol{U}_{\mathrm{sp}} d \mathcal{S}_{n} \\
& +\sqrt{\frac{2 \pi k_{\mathrm{s}}}{i}} \sum_{n=1}^{N} A_{n} \int_{\mathcal{S}_{n}} \frac{e^{i k_{\mathrm{s}}\left(D_{n}+\boldsymbol{r} \cdot \boldsymbol{k}^{\mathrm{inc}}\right)}}{4 \pi \sqrt{D_{n}}} \boldsymbol{U}_{\mathrm{ss}} d \mathcal{S}_{n} .
\end{aligned}
$$

Note that each facet is assigned a vector $\boldsymbol{D}_{n}=\boldsymbol{R}-\boldsymbol{r}_{n}$, where $n$ denotes the facet number. Additional details may be found in [21, although it should be noted that there, the incident waves were assumed to come from a source.

The plane wave amplitude term $A_{n}$ in 21 is defined in one of two ways:

$$
A_{n}=1 \quad \text { (pure); } \quad A_{n}=\exp \left(-x_{n}^{2} / w^{2}\right) \quad \text { (Gaussian tapered), }
$$

depending on the type of plane wave excitation ( $x_{n}$ denotes the centre of the $n$th facet). A pure plane wave excitation is assumed to be of constant amplitude $A_{n}=1$, but with varying phase. For a finite defect, whose length is defined by $L$, an additional refinement to exclude the effect of the tips may be investigated. In this case, a tapered plane wave with a Gaussian amplitude profile is applied, the details of which are explained in detail in Section 4.1. Both the tapered Gaussian and pure plane waves have been analysed for Monte Carlo studies of 200 realisations for two crack lengths $L=8 \mathrm{~mm} \simeq 10 \lambda_{s}$ and $L=4 \mathrm{~mm} \simeq 5 \lambda_{s}$. KA results are compared with FE simulations (described in the next section) and are discussed in what follows, including the differences arising from the inclusion of tip effects. 


\section{Finite Element simulations}

Finite element (FE) methods are used to validate the accuracy of the KA theory via Monte Carlo (MC) simulations. Previous studies [15, 10, 12, have typically used between 50 and 100 rough surface realisations in their FE validations. Here, using the GPU-driven software package Pogo developed by Huthwaite [14, it is possible to run MC simulations of 200 surfaces for domains of size $55 \times 37 \mathrm{~mm}^{2}$ with an incident shear wavelength of $0.8 \mathrm{~mm}$ in under 3.5 hours, using a single GPU. This significant increase in efficiency has enabled the consideration of a greater range of crack lengths, angles of incidence and excitation types than previously reported, as well as the obvious advantage of having larger, and therefore more statistically meaningful, sample sizes of the randomly rough Gaussian surfaces.

All simulations in this article use an aluminium medium with Young's modulus $E=70 \mathrm{GPa}$, density $\rho=2700 \mathrm{~kg} \mathrm{~m}^{-3}$ and Poisson's ratio $\nu=0.33$. The corresponding bulk wave speeds are $190 c_{\mathrm{s}}=3122 \mathrm{~ms}^{-1}$ for shear waves, and $c_{\mathrm{p}}=6198 \mathrm{~ms}^{-1}$ for the longitudinal waves. For each angle of incidence, 18 sets of rough surface profiles are generated for pairs of $\sigma, \lambda_{0}$ using 6 values of RMS $\sigma=\lambda_{\mathrm{s}} / 10, \lambda_{\mathrm{s}} / 8, \lambda_{\mathrm{s}} / 6, \lambda_{\mathrm{s}} / 5, \lambda_{\mathrm{s}} / 4$ and $\lambda_{\mathrm{s}} / 3$, and 3 values of correlation length $\lambda_{0}=2 \lambda_{\mathrm{s}}, \lambda_{\mathrm{s}}$ and $\lambda_{\mathrm{s}} / 2$. For each pair of statistical parameters, 200 random realisations are generated and then, in turn, inserted into a bulk medium of aluminium in one of two ways depending on the type of excitation.

\subsection{Types of plane wave excitation}

A pure plane wave is excited by applying point forces at nodes extending across the entire horizontal breadth of the $2 \mathrm{D}$ domain. In this case, the rough profile is converted to a $2 \mathrm{D}$ defect by appending a lower complementary shadowed line with an appropriate profile. The area bounded by this defect is then excluded from the mesh of linear triangular elements generated by the in-house Pogo mesher, as illustrated in Figure 3 for a case of oblique incidence. The $8 \mathrm{~mm}$ defect has been rotated anticlockwise through $15^{\circ}$ in Figure $3(\mathrm{a})$, simulating oblique incidence of $\theta_{\mathrm{i}}=-15^{\circ}$, as defined in Figure 1

(a)

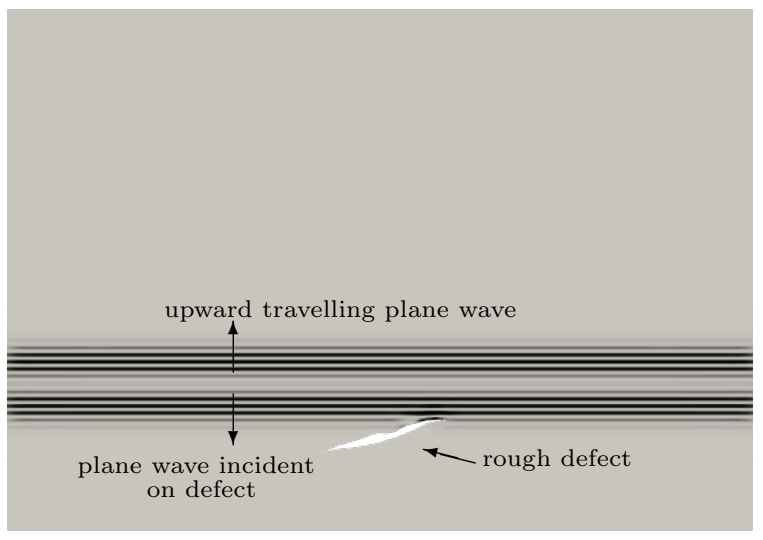

(b)

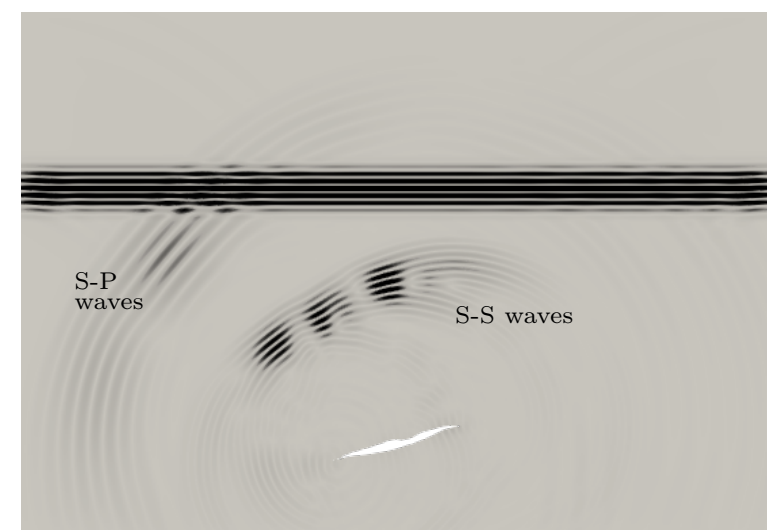

Figure 3: Illustration of pure plane shear wave excitation for a rough surface of length $L=8 \mathrm{~mm}$ characterised by $\sigma=\lambda_{\mathrm{s}} / 6, \lambda_{0}=\lambda_{\mathrm{s}}$. The rough surface has been rotated anticlockwise through $15^{\circ}$ to simulate oblique incidence. (a) Pure plane wave incident on embedded defect. (b) Scattered S-S and S-P waves.

The resulting scattered field is illustrated in Figure 3(b), where the largest amplitudes belong to the upward-travelling incident plane wave and the reflected shear waves. The faster mode-converted longitudinal waves arrive at receivers located in the far-field first, and numerous tip-diffracted and secondary scattered waves are visible. The wave-types are easily discernible from their wavelengths and arrival times. The pure plane wave excitation of Figure 3(a) allows for numerous incident angles by rotating the rough defect, but requires that the $\mathrm{FE}$ domain has absorbing layers on all sides. 
Following preceding literature 2, 22, the Gaussian tapered plane wave has a half beamwidth $w$ satisfying the criteria

$$
10 \lambda_{s} / \pi \leq w \leq L / 5
$$

where the amplitude of excitation forces applied to source nodes within the mesh follows a Gaussian distribution of the form $\exp \left(-x^{2} / w^{2}\right)$. The lower bound in (23) guarantees that the wavefront of the excited wave is plane, and follows 2], whilst the upper bound ensures that tip diffractions may be discounted from the analysis. This upper limit was determined by numerical testing and is similar to the criterion $w \leq L / 6$ given by 22] for longitudinal incidence, the adjustment here arising from the shorter wavelength of shear waves. An example is illustrated in Figure 4, where the rough surface is a $20 \mathrm{~mm}$ section of the backwall. A smoothing function is applied to the final $10 \%$ of each end of the rough surface to facilitate the joining with the smooth part of the backwall. These exterior parts of the rough surface and all of the smooth part of the backwall are not insonified by the incoming plane wave (see Figure 4(a)), ensuring that tip diffraction effects, insufficiently captured by KA theory, do not contribute to errors between the theoretical model and the FE simulations. The scattered field shown in Figure 4(b) resembles that of Figure 3(b), albeit with a

(a)

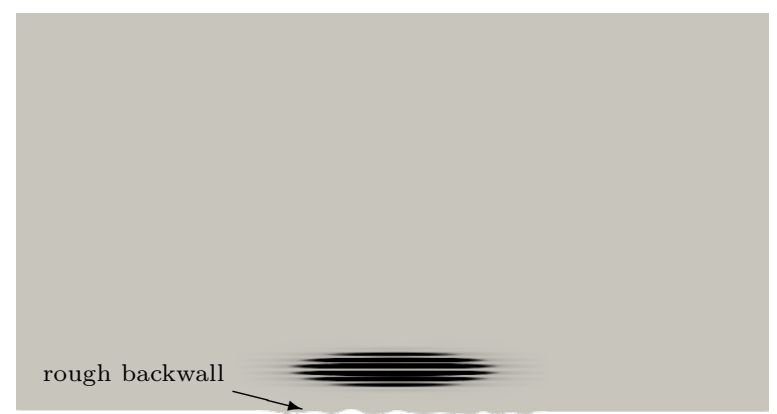

(b)

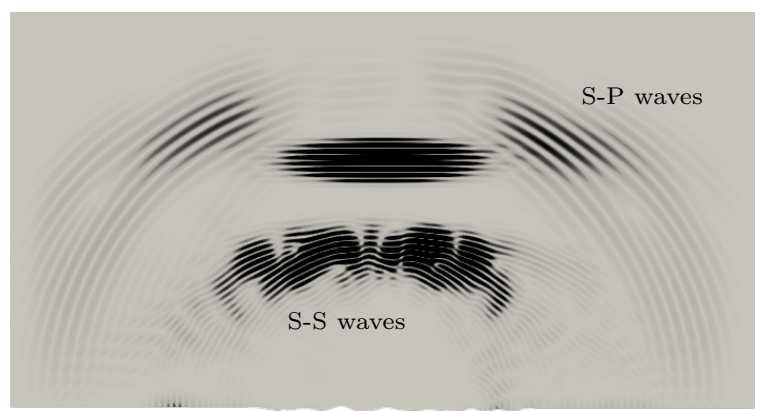

Figure 4: Illustration of tapered plane wave, with a Gaussian amplitude profile, normally incident on a $20 \mathrm{~mm}$ rough surface characterised by $\sigma=\lambda_{\mathrm{s}} / 8, \lambda_{0}=\lambda_{\mathrm{s}}$.(a) Excitation of Gaussian plane wave, prior to incidence on rough backwall. (b) Scattered field comprising shear-shear and shear-longitudinal mode converted waves. Upward travelling Gaussian excitation also shown.

reduction in tip-diffracted signals. The Rayleigh waves observable on the backwall of Figure 4 (b) have arisen from mode-converted surface waves, rather than directly from primary tip scattering.

Note the difference in length of the rough surfaces in Figures 3, 4. For a five-cycle Hann-windowed shear wave with centre frequency of $4 \mathrm{MHz}$ and wavelength $\lambda_{\mathrm{s}} \simeq 0.8 \mathrm{~mm}$ in aluminium, the lower criterion in 23) imposes a minimum half beamwidth of $w \simeq 2.5$, from which a minimum length of $L=5 w>12.5 \mathrm{~mm}$ follows for the upper bound. For a short crack $(4 \mathrm{~mm})$, it is impossible to satisfy the lower and upper limits in (23) simultaneously, and hence we primarily present the results for pure plane wave excitation.

The Gaussian rough backwall set-up has a slightly smaller $2 \mathrm{D}$ domain, $55 \times 28.5 \mathrm{~mm}^{2}$ compared with $55 \times 37 \mathrm{~mm}^{2}$ for the pure plane wave. In both set-ups, the absorbing layer thickness is $5 \mathrm{~mm}>3 \lambda_{\mathrm{p}}[23$ and the far-field receivers are located on the arc of a circle of radius $25 \lambda_{\mathrm{s}}$, with the same centre as the rough surface. The receivers measure the displacements for scattering angles ranging from $-60^{\circ} \leq \theta_{\mathrm{s}} \leq 60^{\circ}$.

\subsection{Signal amplitudes and Hilbert peaks}

Following the work of [10], the ensemble average of the peak of Hilbert transformed scattered signals in the specular direction of the reflected field is used to compare the results of the KA and FE methods. Since the KA calculations of (21) are computed in the frequency domain, these results are converted to the time domain using the input FE signal and the inverse fast Fourier transform (IFFT). Both the FE and KA results are normalised using the specular reflected signal from a flat surface of the same finite length, for normal incidence i.e. $\theta_{\mathrm{i}}=\theta_{\mathrm{s}}=0$. 
(a)

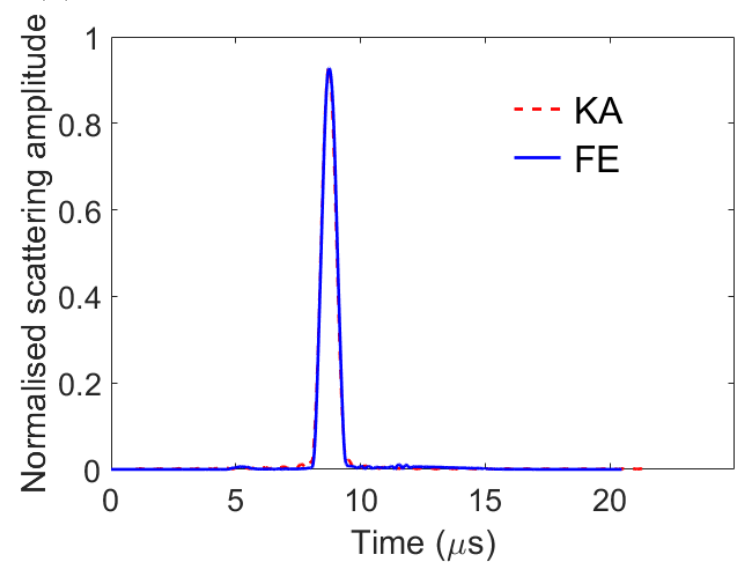

(b)

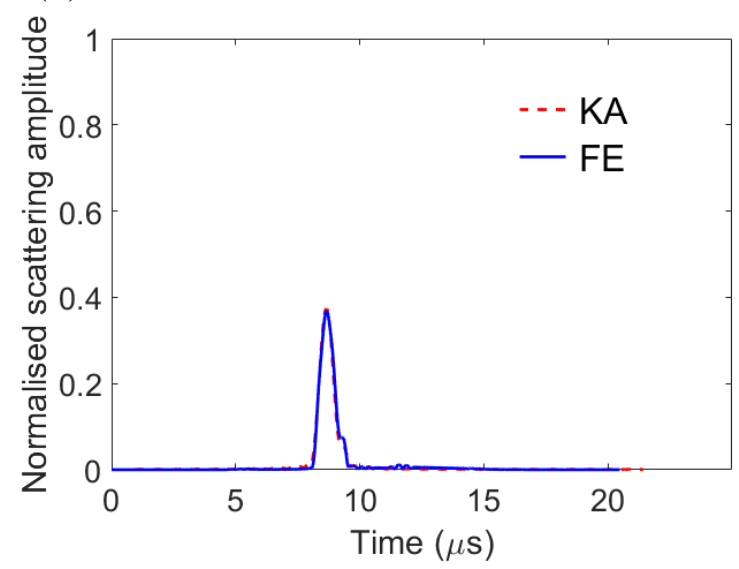

Figure 5: Illustration of Hilbert transformed signal peaks for specular reflection from rough surfaces characterised by $\sigma=\lambda_{\mathrm{s}} / 8, \lambda_{0}=2 \lambda_{\mathrm{s}}$, for normal incidence. Crack length in each case is $L=8 \mathrm{~mm}$. (a) Slightly rough surface (Realisation 1). (b) Slightly rough surface (Realisation 2).

An example is shown in Figure 5 for two cracks of the same length, $L=8 \mathrm{~mm}$, and with the same statistical parameter values, $\sigma=\lambda_{\mathrm{s}} / 8, \lambda_{0}=2 \lambda_{\mathrm{s}}$. The two surfaces may share the same statistical class, but in Figure 5(a), the normalised scattering amplitude at the specular angle is $>90 \%$ of that for the flat defect of the same length, whereas the example of Figure 5(b) has an amplitude of $<40 \%$, indicating large variations in terms of the amplitude. However, both cases show excellent agreement between the KA theory and the FE simulation. It is this agreement that is used to determine the range of validity of KA.

\section{Results}

The ensemble average of the peak of the Hilbert-transformed scattered signals in the specular direction is used as the statistical parameter with which to quantify the range of validity for KA theory for $2 \mathrm{D}$ shear wave incidence. However, all scattered angles within the range $-60^{\circ} \leq \theta_{\mathrm{s}} \leq 60^{\circ}$ are considered. We extract the shear displacement as perpendicular to the propagation direction i.e. $u_{x} \cos \left(\theta_{\mathrm{s}}\right)-u_{z} \sin \left(\theta_{\mathrm{s}}\right)$ (see Figure 11. Examples for $L=8 \mathrm{~mm}$ and $L=4 \mathrm{~mm}$ are shown in Figures 6, 7, respectively.

(a)

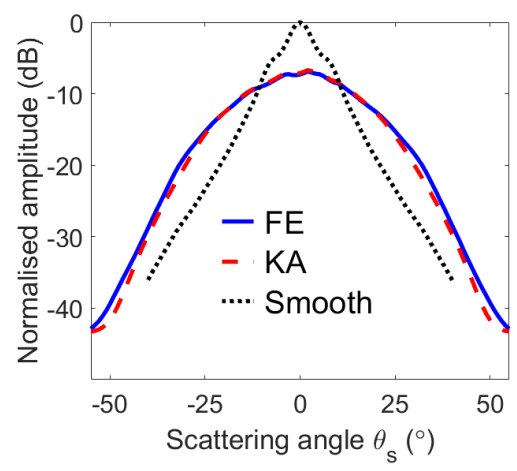

(b)

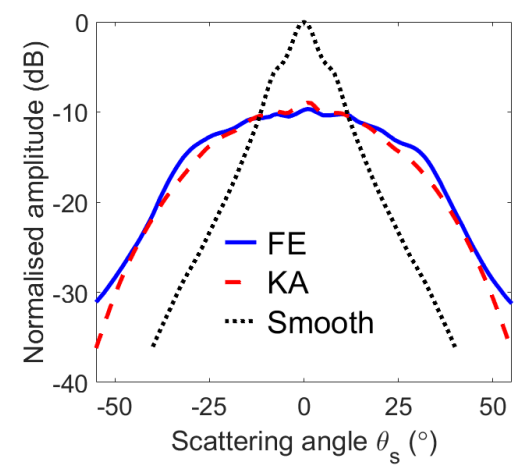

(c)

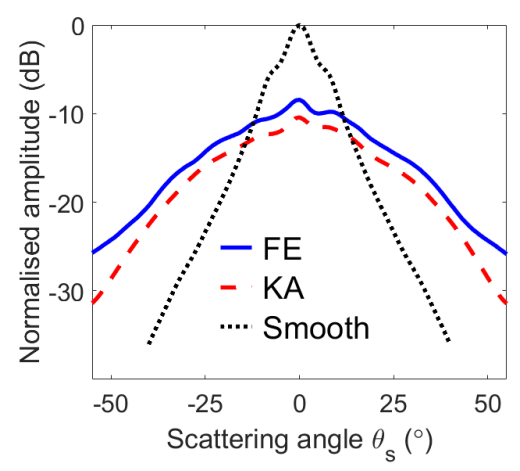

Figure 6: Means of Hilbert transformed signal peaks for scattering angles $-60^{\circ} \leq \theta_{\mathrm{s}} \leq 60^{\circ}$ for rough surfaces of $L=8 \mathrm{~mm}$ characterised by $\sigma=\lambda_{\mathrm{s}} / 6$, for 3 values of $\lambda_{0}$, for normal incidence. (a) $\lambda_{0}=2 \lambda_{\mathrm{s}}$. (b) $\lambda_{0}=\lambda_{\mathrm{s}}$. (c) $\lambda_{0}=\lambda_{\mathrm{s}} / 2$. 
(a)

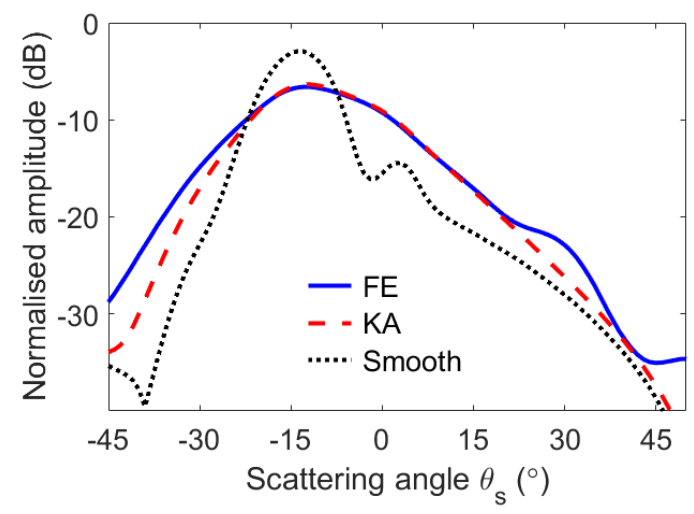

(b)

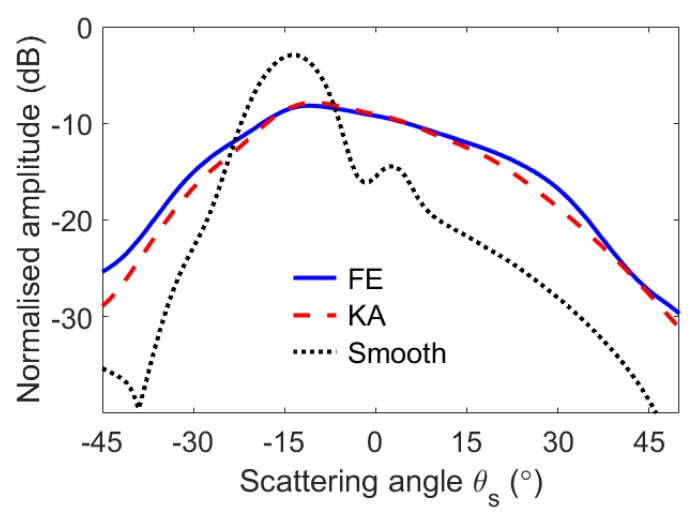

Figure 7: Means of Hilbert transformed signal peaks for scattering angles $-60^{\circ} \leq \theta_{\mathrm{s}} \leq 60^{\circ}$ for rough surfaces of $L=4 \mathrm{~mm}$ characterised by $\sigma=\lambda_{\mathrm{s}} / 8$, for 2 values of $\lambda_{0}$, for incident plane shear wave $\theta_{\mathrm{i}}=-15^{\circ}$. The scattering for a smooth defect of the same length is also shown. (a) $\lambda_{0}=2 \lambda_{\mathrm{s}}$. (b) $\lambda_{0}=\lambda_{\mathrm{s}}$.

The mean values of the Hilbert peaks over all surfaces are obtained for each scattering angle for both $\mathrm{KA}$ and $\mathrm{FE}$, and plotted together with the scattering amplitudes for the flat surface used as the normalising reference. Figure 6 considers normal incidence for 200 rough surfaces characterised by $\sigma=\lambda_{\mathrm{s}} / 6$ and three values of $\lambda_{0}$. As $\lambda_{0}$ is reduced from $2 \lambda_{\mathrm{s}}$ in Figure 6(a) to $\lambda_{\mathrm{s}} / 2$ in Figure 6(c), it is clear that both the magnitude of the specular amplitude, and the agreement between $\mathrm{KA}$ and $\mathrm{FE}$, are reduced. The amplitude is plotted on the $\mathrm{dB}$ scale, and the FE and KA results are shown with, respectively, the solid and dashed curves.

There is a wide angular spread and a significant reduction in magnitude relative to the flat surface. Note the symmetry about the specular peak $\theta_{\mathrm{s}}=0^{\circ}$ throughout Figure 6, which indicates that a sufficient number of realisations has been considered. This observation is particularly clear for the case of normal incidence. A combination of the reduced crack-length and oblique incidence results in more diffuse scattering contributions around the specular angle in Figure 7. mostly due to multiple scattering effects that we discuss in detail later. The major impact from the tips, for the FE method, occurs at the larger scattering angles, rather than near the specular angle, as indicated by the small errors between the KA and FE results for $-20^{\circ} \leq \theta_{\mathrm{s}} \leq 5^{\circ}$ in Figures 7(a) and 7(b), and much larger differences for $\left|\theta_{\mathrm{s}}\right| \geq 30^{\circ}$.

An interesting feature of Figure 6 is that the FE specular amplitude remains roughly the same as the correlation length is reduced from $\lambda_{0}=\lambda_{\mathrm{s}}$ in Figure 6(b) to $\lambda_{\mathrm{s}} / 2$ in Figure 6(c). This is consistent with some findings for longitudinal wave incidence in [24]. Although the combination of $\sigma=\lambda_{\mathrm{s}} / 6, \lambda_{0}=\lambda_{\mathrm{s}} / 2$ is too rough for KA to be formally valid, the differences in scattering amplitude arise not at the specular angle, but in larger contributions at a more diffuse range of scattering angles, with the minimum amplitude being $-25 \mathrm{~dB}$ in Figure 6(c), compared with -30dB in Figure 6(b).

\subsection{Errors between $F E$ and $K A$ results}

We have already discussed a clear example in Figure 6(c), where the KA theory fails to predict the scattered field adequately. In this section, we quantify the error between the KA and FE methods, to determine ranges of validity for KA for the statistical parameters and crack length $L$. Following [10, we use the specular amplitude of the shear-shear mode and define the error of the means, and its standard deviation [25]p.126,326, as follows:

$$
E^{\mathrm{spec}}=\left|\bar{A}^{\mathrm{KA}}-\bar{A}^{\mathrm{FE}}\right|, s\left(E^{\mathrm{spec}}\right)=\sqrt{\frac{s^{2}\left(\bar{A}^{\mathrm{KA}}\right)+s^{2}\left(\bar{A}^{\mathrm{FE}}\right)}{N}} ; \quad \bar{A}=\frac{1}{N} \sum_{n=1}^{N} A_{n}^{*},
$$


where $A_{n}^{*}$ is the specular amplitude obtained from either the FE or KA method for the $n$th realisation, the function $s$ represents the standard deviation of the relevant sample and $N=200$. Mean absolute errors have also been calculated with the definitions:

$$
\bar{E}=\frac{1}{N} \sum_{n=1}^{N} E_{n}=\frac{1}{N} \sum_{n=1}^{N}\left|A_{n}^{\mathrm{KA}}-A_{n}^{\mathrm{FE}}\right|, \quad s(\bar{E})=\sqrt{\frac{1}{N} \sum_{n=1}^{N}\left(E_{n}-\bar{E}\right)^{2}} .
$$

We plot the absolute error of means for $8 \mathrm{~mm}$ cracks in Figure 8(a) for two values of $\theta_{\mathrm{i}}$. The values of correlation length are denoted by marker-type, and $\theta_{\mathrm{i}}=0^{\circ}$ is shown by solid curves, and $\theta_{\mathrm{i}}=-15^{\circ}$, by dashed curves. Standard deviations are indicated by the error bars bracketing each marker point. A solid horizontal line is plotted at $1 \mathrm{~dB}$, which is widely used in practical NDE as a tolerance error [10]. This tolerance error is exceeded for all but two values of $\sigma$ for the case of $\lambda_{0}=\lambda_{\mathrm{s}} / 2$ (denoted by ' + ' markers in Figure $8(\mathrm{a})$ ), indicating that this correlation length is too short for reliable agreement between KA and FE for shear wave incidence once a threshold of $\sigma$ has been passed. Certainly, roughness defined by $\sigma>\lambda_{\mathrm{s}} / 8$ can no longer be accurately accounted for by KA for $\lambda_{0}=\lambda_{\mathrm{s}} / 2$.

(a)

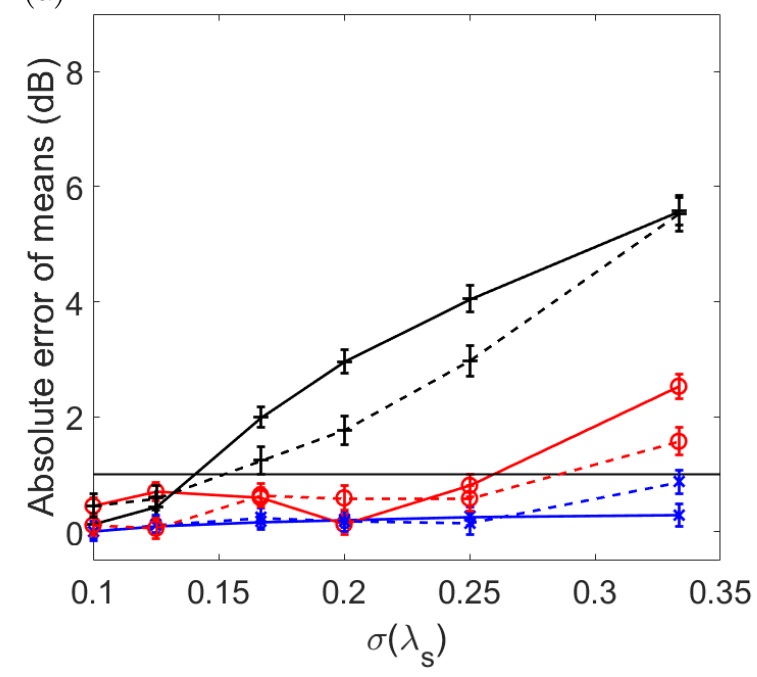

(b)

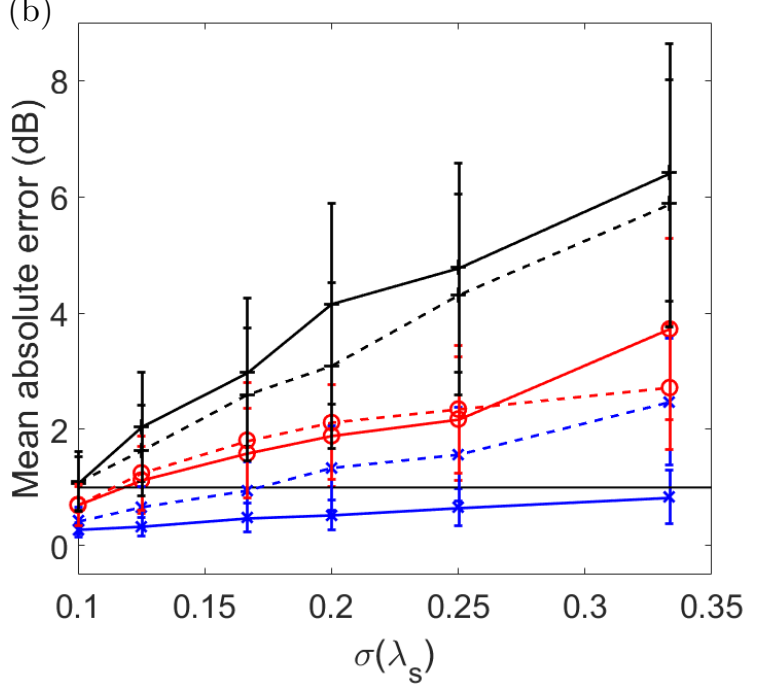

Figure 8: Errors for $\theta_{\mathrm{i}}=0^{\circ}$ (solid curves) and $\theta_{\mathrm{i}}=-15^{\circ}$ (dashed). Marker-type (and colour in online version only) indicates the value of correlation length $\lambda_{0}: 2 \lambda_{\mathrm{s}}$ denoted by ' $\times$ ' (and blue), $\lambda_{\mathrm{s}}$ by ' $\circ$ ' (and red), $\lambda_{\mathrm{s}} / 2$ by ' + ' (and black). Length of crack in all cases is $8 \mathrm{~mm}$. (a) Absolute error of means, see equations (24). (b) Mean absolute error, see equations (25).

A similar observation can be made for the case $\lambda_{0}=\lambda_{\mathrm{s}}$ when $\sigma \geq \lambda_{\mathrm{s}} / 4$, as indicated by the curves with ' $\circ$ ' markers in Figure 8(a). The basic data indicates that somewhere between $\sigma=\lambda_{\mathrm{s}} / 4$ and $\lambda_{\mathrm{s}} / 3$, the tolerance error of $1 \mathrm{~dB}$ is passed. However, detailed analysis indicates that $\sigma=\lambda_{\mathrm{S}} / 4$ is the threshold value, since it is at this value of $\sigma$ that the sign of the error argument changes, i.e. the FE method begins to predict larger amplitudes than KA, whereas for lower $\sigma$ values, it is the KA method that overpredicts the specular amplitudes. This is illustrated in the example of Figure 6, where the dashed KA curve in Figure 6(b) exceeds the solid curve at $\theta_{\mathrm{s}}=0$, but in Figure 6(c), the opposite is true for $\lambda_{0}=\lambda_{\mathrm{s}} / 2$. The transitional behaviour for $\lambda_{0}=\lambda_{\mathrm{s}}$ at $\sigma=\lambda_{\mathrm{s}} / 4$ is repeated for $\lambda_{0}=\lambda_{\mathrm{s}} / 2$ at $\sigma=\lambda_{\mathrm{s}} / 8$. These thresholds indicate when the validity of the approximation begins to break down. In both cases, local and global effects are simultaneously contributing to errors between FE and KA.

It is clear that the threshold of validity is reached at much lower levels of roughness when compared with longitudinal incidence [10]. The data of Figure 8(a) is reminiscent of the results for correlation lengths 
$\lambda_{\mathrm{p}} / 2, \lambda_{\mathrm{p}} / 4$ in Figure 10 of [10]. In real terms the corresponding correlation lengths are roughly equal, but everything is measured in terms of incident wavelengths (shear here, longitudinal in [10]). Thus, the corresponding $\sigma$ values differ by a factor of 2 . In essence, comparison of results for $\lambda_{0}=\lambda_{\mathrm{s}}$ and $\lambda_{0}=\lambda_{\mathrm{p}} / 2$ may be considered as a doubling of roughness, and so the range of validity for KA theory for shear wave incidence is approximately half that for longitudinal incidence.

The mean absolute error (MAE) results calculated using equations (25), and illustrated in Figure 8(b) also support this qualitative comparison of shear and longitudinal incidence. For all $\sigma$ values of $\lambda_{0}=\lambda_{\mathrm{s}}$ (bar $\sigma=\lambda_{\mathrm{s}} / 10$ ), the MAE results exceed the $1 \mathrm{~dB}$ tolerance in Figure 8(b). A similar observation was made for $\lambda_{0}=\lambda_{\mathrm{p}} / 2$ in Figure 6(f) of [10, where results for $\sigma \geq \lambda_{\mathrm{p}} / 8$ are similar to those shown for $\sigma \geq \lambda_{\mathrm{s}} / 8$ for the ' $\circ$ ' markers in Figure $8(\mathrm{~b})$.

\section{Discussion of results}

The results of Section 5 indicate that KA theory becomes invalid for shear wave incidence at a level of roughness half that for longitudinal incidence. In this section, we explain the reasons for this significant difference and the mechanisms that lead to the theory becoming insufficiently accurate. As stated by [10] for the case of longitudinal incidence, both the RMS $\sigma$, and the correlation length $\lambda_{0}$, are important, but the dominant factor for shear wave incidence appears to be the latter parameter, as illustrated in Figure 6.

Thorsos [7] made a similar observation for the case of acoustic wave scattering, and this was also reported by [1, who explained that the correlation length only affects the diffuse field, since for scattering around the specular direction, the relative phases of the waves depend only on variations in height, i.e. $\sigma$, but away from the specular direction, the relative phases depend on both height and lateral separation, i.e. $\lambda_{0}$. However, it is important to note that the diffuse field also makes a contribution in the specular direction. The specular scattering is not solely the coherent part of the field, but a sum of coherent and diffuse parts. Indeed, for many of the rough surfaces considered in this paper, the dominant part of the field is the diffuse part, even in the specular direction.
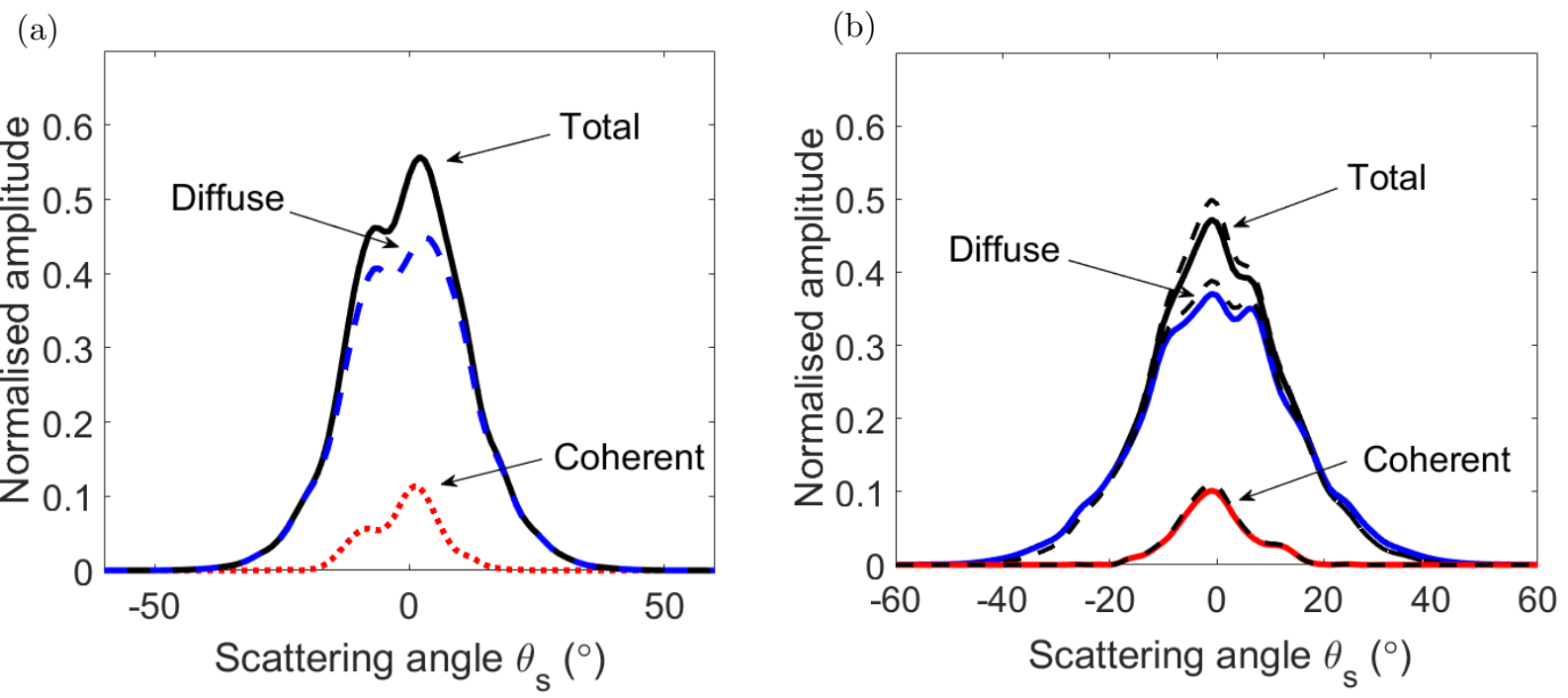

Figure 9: Means of Hilbert transformed signal peaks for scattering angles $-60^{\circ} \leq \theta_{\mathrm{s}} \leq 60^{\circ}$ for rough surfaces characterised by $\sigma=\lambda_{\mathrm{s}} / 8$ for normally incident plane shear wave $\theta_{\mathrm{i}}=0^{\circ}$. Crack length in each case is $L=8 \mathrm{~mm}$. (a) $\lambda_{0}=2 \lambda_{\mathrm{s}}$. Only FE results are shown. (b) $\lambda_{0}=3 \lambda_{\mathrm{s}} / 2$. Solid lines represent FE results, dashed lines represent KA method.

The Monte Carlo simulations use the following formulae to calculate the total and coherent amplitudes 
for the FE and KA methods:

$$
A_{\mathrm{KA} / \mathrm{FE}}^{\mathrm{t}}=\frac{1}{N} \sum_{n=1}^{N}\left|\boldsymbol{u}_{n}^{\mathrm{sc}}\left(\theta_{s}\right)\right| ; \quad A_{\mathrm{KA} / \mathrm{FE}}^{\mathrm{c}}=\left|\frac{1}{N} \sum_{n=1}^{N} \boldsymbol{u}_{n}^{\mathrm{sc}}\left(\theta_{s}\right)\right| .
$$

The diffuse field is the difference of the two:

$$
A_{\mathrm{KA} / \mathrm{FE}}^{\mathrm{d}}=A_{\mathrm{KA} / \mathrm{FE}}^{\mathrm{t}}-A_{\mathrm{KA} / \mathrm{FE}}^{\mathrm{c}} .
$$

An example is shown in Figure 9(a) for a low roughness case with statistical parameters $\lambda_{0}=2 \lambda_{\mathrm{s}}, \sigma=\lambda_{\mathrm{s}} / 8$ for normal incidence using a tapered Gaussian plane wave, to discount any contributions from the tips of the defects. Only the FE results for 200 surfaces are shown in Figure 9(a), since the error between the FE and KA methods for this case is minimal. The specular direction is $\theta_{\mathrm{s}}=0$, and the results show that the mean normalised total amplitude is $55 \%$, with a $10 \%$ coherent contribution, and a $45 \%$ diffuse contribution.

In Figure 9(b), 200 new surfaces were generated with the correlation length reduced to $\lambda_{0}=3 \lambda_{\mathrm{s}} / 2$. As expected for the rougher case, the mean total amplitude in the specular direction falls, but the coherent part of the field is affected minimally. The reduction in correlation length not only reduces the amplitude of the diffuse field contribution, from $45 \%$ to $37 \%$, but also leads to an increased error between the FE (solid) and KA (dashed) predictions.

Most preceding studies of elastic wave scattering by rough surfaces have illustrated and tested models for longitudinal wave incidence. One previous study provided results for vertically polarised shear waves (SV) incident upon randomly rough $2 \mathrm{D}$ surfaces [12. The statistical roughness data of [12] was obtained using a Hann window autocorrelation approach, rather than the Gaussian profile adopted here. An alternative to the standard deviation of the height data $(\mathrm{rms} \sigma)$ and a frequency of $10 \mathrm{MHz}$ were used by [12, so it is not easy to compare results directly with those presented here but there are some general observations that appear to be consistent.

(a)

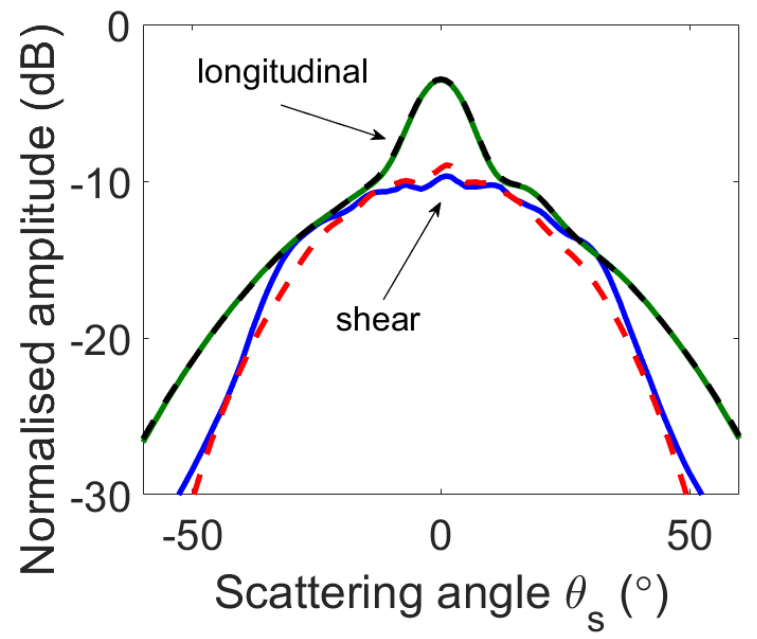

(b)

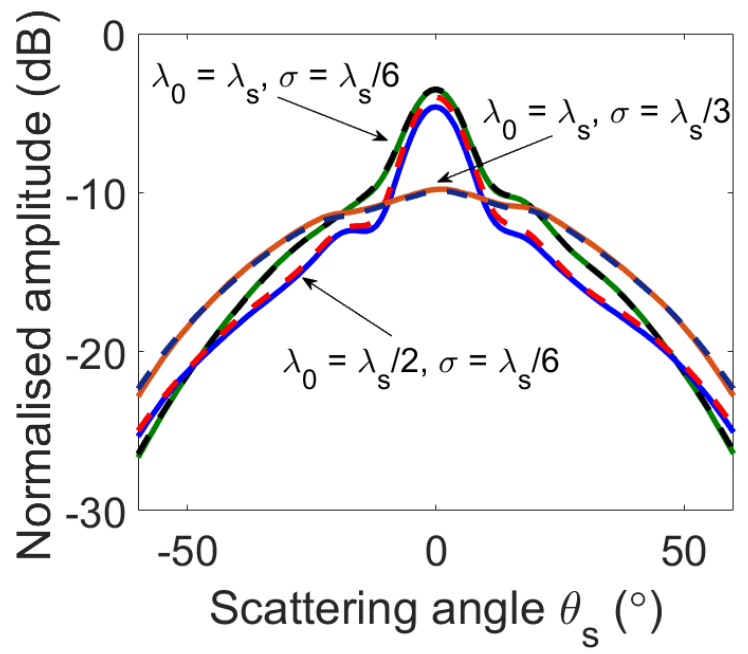

Figure 10: Means of Hilbert transformed signal peaks for scattering angles $-60^{\circ} \leq \theta_{\mathrm{s}} \leq 60^{\circ}$ for normal incidence and $L=8 \mathrm{~mm}$. Solid lines represent FE results, dashed lines represent KA method. (a) Shear wave vs longitudinal wave for normal incidence and $\lambda_{0}=\lambda_{\mathrm{s}}, \sigma=\lambda_{\mathrm{s}} / 6$. (b) Longitudinal incidence with $\sigma$ and $\lambda_{0}$ varied from part(a).

We compare shear and longitudinal incidence directly in Figure 10. The surfaces characterised by $\lambda_{0}=\lambda_{\mathrm{s}}$, $\sigma=\lambda_{\mathrm{s}} / 6$ in Figure 6(b) are insonified by longitudinal waves in Figure 10(a). The amplitude at the specular scattering angle is much lower for the shear-shear modes than for the longitudinal-longitudinal modes. 
Equally strikingly, there is clear error between the FE and KA results for shear incidence, but for longitudinal incidence for the same set of surfaces, and for the whole range of scattering angles, there is excellent agreement.

It is true that the surfaces are defined using shear wavelengths, and are therefore equivalent to $\lambda_{0}=\lambda_{\mathrm{p}} / 2$, $\sigma=\lambda_{\mathrm{p}} / 12$. The relative reduction in roughness $\sigma$ leads to a larger amplitude for the P-P modes compared with the S-S modes, but the extent of the difference in KA-FE agreement between the two types of incident wave is less predictable.

Figure 10(b) illustrates both the robustness of the KA-FE agreement for longitudinal incidence and the importance of correlation length. The surfaces of Figure 10(a) are shown again for longitudinal incidence, along with the cases of doubling $\sigma$, and halving $\lambda_{0}$. The excellent agreement between KA and FE results is retained for all scattering angles. A small reduction in specular amplitude and a visible disagreement in KA and FE results are observed for halving the correlation length. Doubling $\sigma$ leads to a significant decrease in specular amplitude, but has no impact on KA-FE agreement.

Thus, KA-FE agreement is better and more robust for longitudinal incidence. In addition, for equivalent changes in either $\sigma$ or $\lambda_{0}$ from a reference pair of the statistical parameters, the correlation length has more impact on agreement for both longitudinal and shear wave incidence, but with a greater effect for shear waves (compare Figures $10(\mathrm{~b})$ and 6(b) 6(c)).

\subsection{Multiple scattering}

Although KA is precise for infinite smooth surfaces, it is an approximation for finite rough defects with limitations associated with both length and roughness. The theory does not conserve energy [1], therefore neglecting both surface waves and multiple scattering, and does not model tip diffraction effects well owing to the infinite tangential plane assumption. Surface roughness affects the accuracy of KA locally, via the fundamental assumption that the derivation of the local surface from flat is small compared with the incoming wavelength, and globally, via multiple scattering and surface shadowing effects.

An instructive example is Figure 9(b), where the use of normal incidence rules out shadowing, the Gaussian tapered plane wave excludes tip effects and the roughness setting $\lambda_{0}=3 \lambda_{\mathrm{s}} / 2, \sigma=\lambda_{\mathrm{s}} / 8$ guarantees that the radius of curvature condition derived by [5] and described in detail by [1]:

$$
k_{\mathrm{s}} r_{c} \cos ^{3} \theta_{i} \gg 1, \quad r_{c}=-\left[1+\left(\frac{\mathrm{d} h}{\mathrm{~d} x}\right)^{2}\right]^{3 / 2}\left(\frac{\mathrm{d}^{2} h}{\mathrm{~d} x^{2}}\right)^{-1},
$$

is not violated for any of the facets for all surfaces. Fundamentally, the radius of curvature of the rough surface for each facet is restricted relative to the incident wavelength, with the extent of this restriction being dependent on the angle of incidence. Note that the condition on the radius of curvature $r_{c}$ simplifies to $k_{\mathrm{s}} r_{c} \gg 1$ for normal incidence, and that 28 is more sensitive to longitudinal incidence since $k_{\mathrm{s}}>k_{\mathrm{p}}$.

The small errors that we see around the specular scattering direction in Figure 9(b) must therefore arise from multiple scattering or surface wave effects. It is straightforward to define thresholds for these global effects using the reflected angles for the scattered waves. For normal incidence illustrated in Figure 11 . incident longitudinal waves will not mode-convert to surface waves (this would require a facet with infinite slope), and multiple scattering becomes significant when

$$
\alpha_{\mathrm{pp}} \geq \pi / 4
$$

For incident shear waves, since the mode-converted waves have a larger scattering angle, the respective threshold conditions for multiple scattering and surface wave mode conversion, are

$$
\alpha_{\mathrm{ss}}+\alpha_{\mathrm{sp}} \geq \pi / 2, \quad \alpha_{\mathrm{sp}} \geq \pi / 2 .
$$

For the calculation of (21) for each Monte Carlo simulation, the multiple scattering and surface wave conditions are computed for each facet of each realisation, and an indication of the these global effects is obtained. Figure 12 illustrates this concept for the two pairs discussed in Section 5.1 Figure 8 as transitional states for the validity of KA theory. 


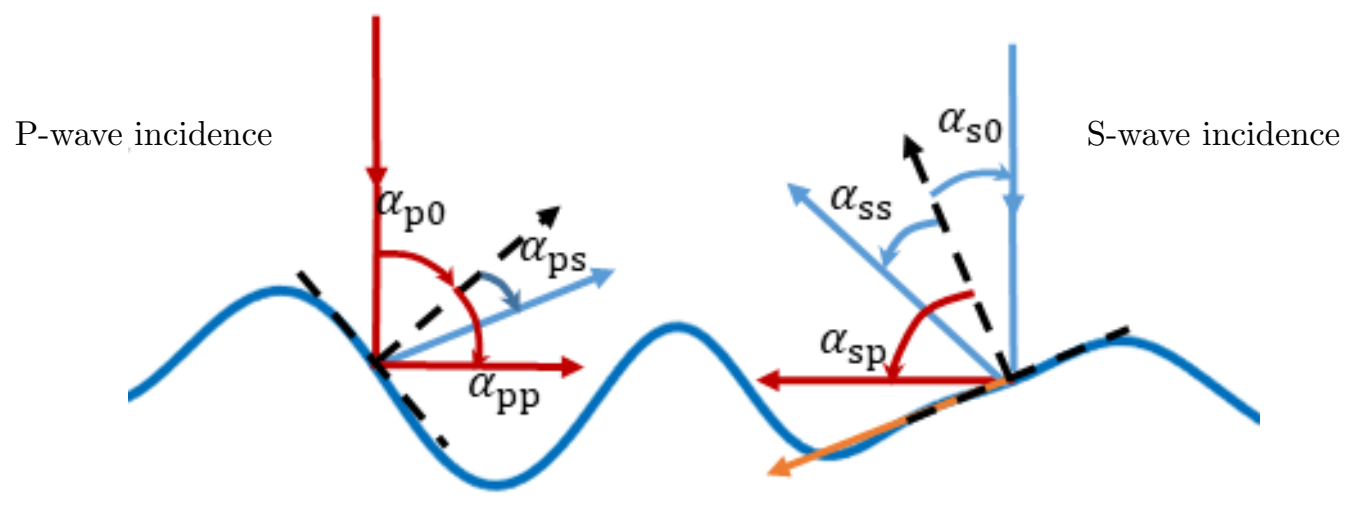

Figure 11: Multiple scattering, surface wave conditions for longitudinal and shear wave normal incidence.

In Figure 12(a), we consider the case with $\lambda_{0}=\lambda_{\mathrm{s}}$ and $\sigma=\lambda_{\mathrm{s}} / 4$, comparing shear with longitudinal incidence. The vertical axis gives the percentage of the total number of facets for which multiple scattering, denoted by ' $\circ$ ', and surface wave mode conversion, denoted by ' $x$ ', may occur for each realisation. The horizontal solid and dashed lines give the mean percentage per realisation for, respectively, multiple scattering and surface wave conversion.

Virtually all realisations experience some multiple scattering or surface wave effects for this level of roughness for shear waves (left), whereas the longitudinal waves (right) produce little or no global effects that may reduce the KA-FE agreement. A similar result is obtained for $\lambda_{0}=\lambda_{\mathrm{S}} / 2$ and $\sigma=\lambda_{\mathrm{S}} / 8$ in Figure $12(\mathrm{~b})$, although with a slightly higher mean percentage for both multiple scattering (15.4\% compared with $14.8 \%$ ), and surface wave effects (9.8\% compared with $9.4 \%$ ), and noticeably fewer outliers for both shear and longitudinal incidence. The difference in terms of outliers for multiple scattering is explained by the halving of the roughness parameter $\sigma$ in Figure $12(\mathrm{~b})$.

The contrast in the mean percentages for shear/longitudinal incidence is striking, being around 30 times larger for shear incidence. It is also important to note that these two shear examples are within the $1 \mathrm{~dB}$ error bound of Figure 8(a), i.e. the KA-FE agreement is deemed satisfactory for the examples of Figure 12 However, it is clear that multiple scattering and surface wave effects have a big impact on KA-FE agreement for shear incidence, and are the explanation for the errors that appear for shear waves at much lower roughness levels than for longitudinal incidence.

We also conclude that it is multiple scattering and surface wave conversion that lead to KA theory overestimating specular amplitudes compared with the true values for $\lambda_{0} \geq \lambda_{\mathrm{s}}$. KA theory fails to account for interference effects correctly at these levels of roughness. For corresponding roughness with longitudinal incidence, there is little or no multiple scattering, and no surface waves for normal incidence. As a result, little or no error was obtained in the previous literature [10].

\subsection{Range of validity for KA for shear wave incidence}

The dominant contributions to KA-FE error for normally incident shear wave are multiple scattering and surface waves. Oblique incidence introduces additional shadowing and tip diffraction contributions. Local effects of surface curvature also play a role as correlation length is reduced. For a given $\lambda_{0}$, as $\sigma$ is increased, the KA-FE error changes sign at a certain threshold. From this point, the FE method predicts higher values of the specular amplitude, and KA theory becomes insufficient. Analysis of individual facets for different realisations reveals when the criteria for multiple scattering and local surface gradient become relevant, and specific critical thresholds are thereby defined.

Column 1 of Table 1 gives $\lambda_{0}$ values, and column 2 specifies the critical value of $\sigma$ for which the error changes sign and FE predicts higher amplitudes than KA theory for all subsequent increases in $\sigma$. This 
(a)
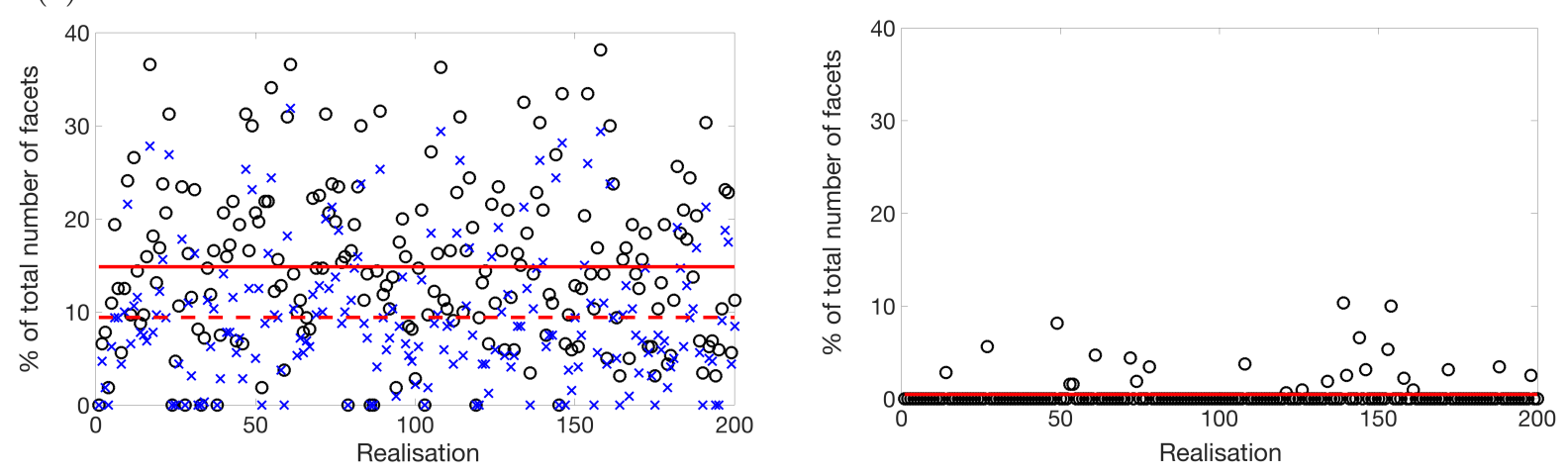

(b)
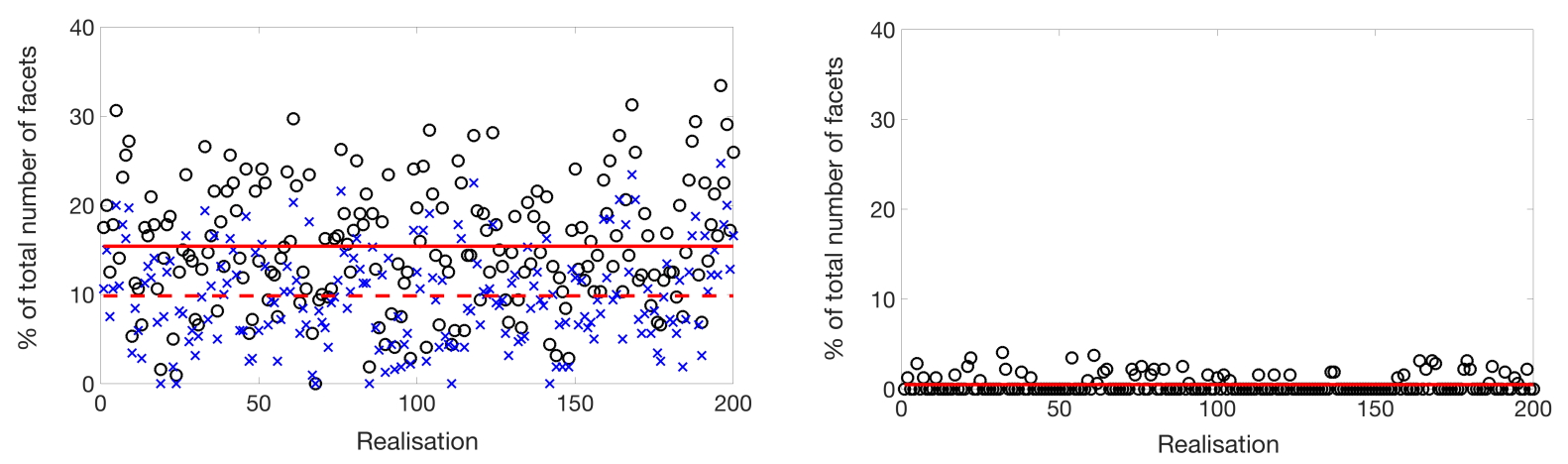

Figure 12: Multiple scattering effects for shear waves (LHS) versus longitudinal waves (RHS) for the same set of surfaces. The markers ' 0 ' indicate multiple scattering, ' $x$ ' indicate surface waves, the solid and dashed lines represent the means for, respectively, multiple scattering and surface waves. Normal incidence for $L=8 \mathrm{~mm}$. (a) $\lambda_{0}=\lambda_{\mathrm{s}}, \sigma=\lambda_{\mathrm{s}} / 4$. (b) $\lambda_{0}=\lambda_{\mathrm{s}} / 2, \sigma=\lambda_{\mathrm{s}} / 8$.

transition signifies the roughness at which sufficiently small errors $(<1 \mathrm{~dB})$ arising from global multiple scattering or surface wave effects grow too large and invalidate KA. Interestingly, for $\lambda_{0}=\lambda_{\mathrm{s}}$, this $\sigma$ value also indicates when the curvature of facets criterion (28) becomes statistically relevant. The significance of this coincidence is that for $\lambda_{0}<\lambda_{\mathrm{s}}$, low values of $\sigma$ introduce local surface errors ahead of the multiple scattering threshold. However, they produce only marginally elevated KA values until multiple scattering becomes the dominant factor, and KA validity breaks down.

An example is shown in Figure 13 , where the statistical roughness parameters are $\lambda_{0}=\lambda_{\mathrm{s}} / 2, \sigma=\lambda_{\mathrm{s}} / 12$ for normal incidence and $L=8 \mathrm{~mm}$, i.e. somewhere between the second and third columns of row 3 in Table 1. Figure 13(a) shows the Hilbert peak amplitudes and the specular direction shows very small error. The explanation appears in Figure 13.(b), where the local radius of curvature criterion (indicated by the ' $x$ ' markers) appears statistically more significant than the multiple scattering effects (' $\circ$ ' markers), with a mean of $8.7 \%$ compared with $3.3 \%$. If these percentages were the other way round, the error would be fairly significant (compare with Figure 12), emphasising that multiple scattering is more important for shear incidence than local surface curvature.

Thus, the range of validity for KA for $L \geq 5 \lambda_{\mathrm{s}}$ is determined using the ratio $\xi=\lambda_{0} / \sigma$ by defining the critical transitional ratio as

$$
\xi_{\mathrm{t}}^{\mathrm{s}}=\frac{\lambda_{0}}{\sigma}=4
$$

such that for $\xi \leq \xi_{\mathrm{t}}^{\mathrm{s}}$, KA theory becomes insufficiently valid for rough surface scattering. A strict greater than relationship is assumed for validity since oblique incidence introduces small differences related to tips and shadowing. 


\begin{tabular}{c|c|c}
\hline$\lambda_{0}\left(\lambda_{\mathrm{s}}\right)$ & $\sigma\left(\lambda_{\mathrm{s}}\right)$ for multiple scattering & $\sigma\left(\lambda_{\mathrm{s}}\right)$ for local curvature \\
\hline 2 & $1 / 2$ & 1 \\
\hline 1 & $1 / 4$ & $1 / 4$ \\
\hline $1 / 2$ & $1 / 8$ & $1 / 16$ \\
\hline
\end{tabular}

Table 1: Thresholds for multiple scattering effects and local radius of curvature conditions for shear waves

(a)

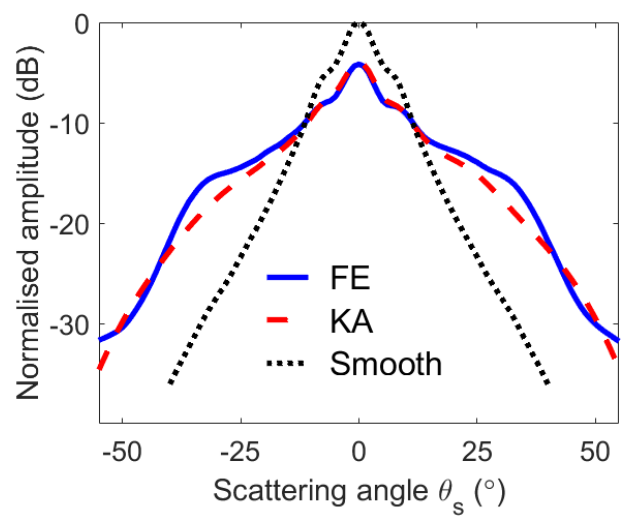

(b)

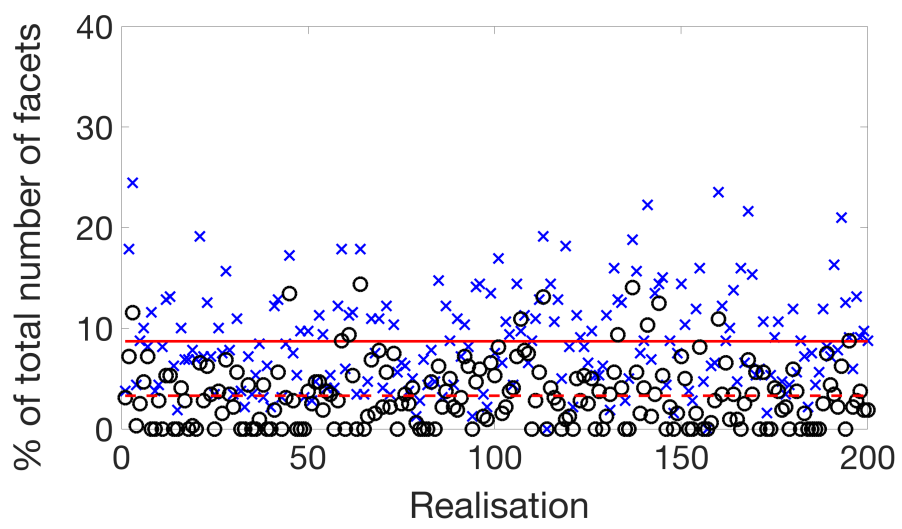

Figure 13: Rough surfaces characterised by $\sigma=\lambda_{\mathrm{s}} / 12$, for $\lambda_{0}=\lambda_{\mathrm{s}} / 2$, for normal incidence. Crack length in each case is $L=8 \mathrm{~mm}$. (a) Means of Hilbert transformed signal peaks for scattering angles $-60^{\circ} \leq \theta_{\mathrm{s}} \leq 60^{\circ}$. (b) Multiple scattering denoted by ' $\circ$ ', radius of curvature condition by ' $\times$ ' with their respective means shown by dashed and straight lines.

The dependence on the column 2 thresholds of Table 1, rather than column 3, highlights the significant difference between shear and longitudinal incidence. As one may expect, the curvature condition (28) is more important for longitudinal incidence, and the importance of multiple scattering and the role of correlation length are enhanced for shear wave incidence, due to the larger reflection angles and surface wave mode conversion. The difference is illustrated further in Figure 14, where for $\lambda_{0}=2 \lambda_{\mathrm{s}}$ and $\lambda_{\mathrm{s}}$, multiple scattering is twice as prevalent for shear incidence than for longitudinal incidence.

\section{Conclusions}

The scattering of vertically polarised shear waves (SV) incident upon randomly rough 2D surfaces brings important results for industrial applications. Firstly, agreement between KA and FE results deteriorates at lower levels of roughness than for longitudinal incidence, and secondly, the KA over-predicts scattering compared with FE predictions for a fixed correlation length until a certain roughness threshold is passed.

We show what this threshold is, and for the first time, explain why and how shear wave scattering differs from longitudinal wave scattering by a rough surface in 2D. Crucially, using Pogo 14 we are able to validate the KA predictions using a minimum of $200 \mathrm{FE}$ realisations, a far greater number than the 50 realisations assessed by each of [15, 10] and the 100 of [12. The greater sample size was used to analyse the multiple scattering effects, shown to be enhanced for shorter correlation lengths, that have previously been difficult to quantify [10. In this way, a reliable and explicable range of validity for KA for shear wave incidence was established to be much smaller than previously anticipated; KA validity breaks down for shear incidence at half the roughness observed for longitudinal incidence.

An additional key feature is surface wave mode conversion, a phenomenon that becomes relevant for longitudinal incidence only when roughness is very high and in cases of grazing angles. In fact, it is impossible 


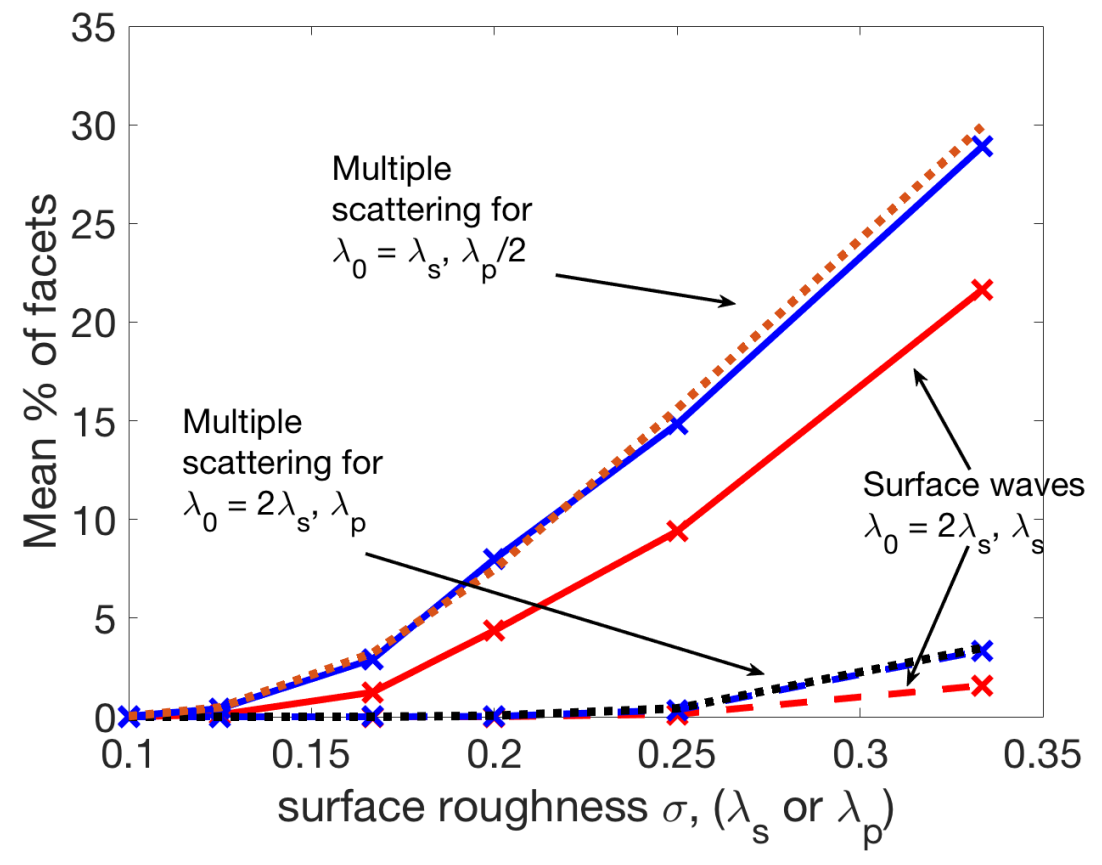

Figure 14: Multiple scattering effects for shear waves versus longitudinal waves (dotted lines) for $\lambda_{0}=2 \lambda_{\mathrm{s}}$ (dashed) and $\lambda_{0}=\lambda_{\mathrm{s}}$ (solid), for normal incidence. Surface wave effects also shown for shear waves. Crack length in each case is $L=8 \mathrm{~mm}$.

for normally incident longitudinal waves to mode-convert to surface waves.

Oblique incidence was considered and the results were consistent with the illustrated normal incidence examples. Small differences arose from tip diffraction and surface shadowing. The length of the defect also brings some additional effects. Results for $L=4 \mathrm{~mm}$ and $L=8 \mathrm{~mm}$ are broadly consistent, with two points of interest. For small values of $\sigma$, the errors are marginally larger for the smaller length, stemming from inadequate allowance for the tip diffraction using KA theory. The opposite occurs as $\sigma$ increases since then the multiple scattering effects dominate, but the reduced length results in a small reduction of the errors associated with multiple scattering.

The results of this paper will be used for a state-of-the-art technique to derive analytical elastodynamic formulae for the expected scattering of elastic shear waves incident upon randomly rough surfaces. The key idea is a stationary phase analysis of Kirchhoff approximation (KA) expressions [8]; the range of validity for KA for shear waves established here is required for the technical derivations. It is also notable that it is possible to extend the KA approach to include secondary scattering [26, 27] and thereby potentially increase the range of validity.

\section{Acknowledgements}

The authors gratefully acknowledge the support of the UK Engineering and Physical Sciences Research Council (EPSRC) through the Grant reference number EP/P01951X/1. FS and PH acknowledge the support of the EPSRC through the Fellowship reference number EP/M020207/1 and RVC acknowledges the support of a Leverhulme Trust Research Fellowship.

\section{References}

[1] J. Ogilvy, Theory of wave scattering from random rough surfaces, CRC Press, 1991. 
[2] C. A. Schultz, M. N. Toksöz, Enhanced backscattering of seismic waves from a highly irregular, random interface: P-SV case, Geophysical Journal International 117 (3) (1994) 783-810.

[3] J. O. Robertsson, R. Laws, C. Chapman, J.-P. Vilotte, E. Delavaud, Modelling of scattering of seismic waves from a corrugated rough sea surface: a comparison of three methods, Geophysical Journal International 167 (1) (2006) 70-76.

[4] A. Maznev, Boundary scattering of phonons: Specularity of a randomly rough surface in the small-perturbation limit, Physical Review B 91 (13) (2015) 134306.

[5] F. Bass, I. Fuks, Wave scattering from statistically rough surfaces, Pergamon Oxford, UK, 1979.

[6] J. Ogilvy, Theoretical comparison of ultrasonic signal amplitudes from smooth and rough defects, NDT \& E International 19 (1986) 371-385.

[7] E. I. Thorsos, The validity of the Kirchhoff approximation for rough surface scattering using a Gaussian roughness spectrum, The Journal of the Acoustical Society of America 83 (1) (1988) 78-92.

[8] F. Shi, M. Lowe, X. Xi, R. Craster, Diffuse scattered field of elastic waves from randomly rough surfaces using an analytical Kirchhoff theory, Journal of the Mechanics and Physics of Solids 92 (2016) 260-277.

[9] P. Beckmann, A. Spizzichino, The scattering of electromagnetic waves from rough surfaces, Artech House Inc, Norwood, MA, 1987.

[10] F. Shi, W. Choi, M. Lowe, E. Skelton, R. Craster, The validity of Kirchhoff theory for scattering of elastic waves from rough surfaces, Proceedings of the Royal Society of London A: Mathematical, Physical and Engineering Sciences 471 (2178) (2015) 20140977.

[11] J. Zhang, B. W. Drinkwater, P. D. Wilcox, Effect of roughness on imaging and sizing rough crack-like defects using ultrasonic arrays, IEEE transactions on ultrasonics, ferroelectrics, and frequency control 59 (5) (2012) 939-948.

[12] R. A. Roberts, The effect of crack morphology on ultrasonic response, in: AIP Conference Proceedings, Vol. 1430, AIP, 2012, pp. 150-157.

[13] P. D. Wilcox, A. Velichko, Efficient frequency-domain finite element modeling of two-dimensional elastodynamic scattering, The Journal of the Acoustical Society of America 127 (1) (2010) 155-165.

[14] P. Huthwaite, Accelerated finite element elastodynamic simulations using the GPU, Journal of Computational Physics 257 (2014) 687-707.

[15] J. Zhang, B. W. Drinkwater, P. D. Wilcox, Longitudinal wave scattering from rough crack-like defects, IEEE transactions on ultrasonics, ferroelectrics, and frequency control 58 (10) (2011) 2171-2180.

[16] J. Ogilvy, Computer simulation of acoustic wave scattering from rough surfaces, Journal of Physics D: Applied Physics 21 (2) (1988) 260-277.

[17] J. Ogilvy, J. Foster, Rough surfaces: Gaussian or exponential statistics?, Journal of Physics D: Applied Physics 22 (9) (1989) 1243-1251.

[18] W. Choi, F. Shi, M. J. Lowe, E. A. Skelton, R. V. Craster, W. L. Daniels, Rough surface reconstruction of real surfaces for numerical simulations of ultrasonic wave scattering, NDT \& E International 98 (2018) 27-36.

[19] J. Achenbach, Wave Propagation in Elastic Solids, North-Holland Publishing Company/American Elsevier, 1973.

[20] J. D. Achenbach, A. K. Gautesen, H. McMaken, Ray methods for waves in elastic solids: with applications to scattering by cracks, Pitman advanced publishing program, 1982.

[21] J. Ogilvy, I. Culverwell, Elastic model for simulating ultrasonic inspection of smooth and rough defects, Ultrasonics 29 (6) (1991) 490-496.

[22] F. Shi, M. Lowe, R. Craster, Diffusely scattered and transmitted elastic waves by random rough solid-solid interfaces using an elastodynamic Kirchhoff approximation, Physical Review B 95 (21) (2017) 214305.

[23] P. Rajagopal, M. Drozdz, E. A. Skelton, M. J. Lowe, R. V. Craster, On the use of absorbing layers to simulate the propagation of elastic waves in unbounded isotropic media using commercially available finite element packages, NDT \& E International 51 (2012) 30-40.

[24] J. R. Pettit, A. E. Walker, M. J. Lowe, Improved detection of rough defects for ultrasonic nondestructive evaluation inspections based on finite element modeling of elastic wave scattering, IEEE Transactions on ultrasonics, ferroelectrics, and frequency control 62 (10) (2015) 1797-1808.

[25] G. Udny Yule, M. Kendall, An introduction to the theory of statistics, no. 14th ed, Charles Griffin \& Co. Ltd., London, 1950.

[26] J.-J. Embrechts, Simulation of first and second order scattering by rough surfaces with a sound ray formalism, Journal of Sound and Vibration 229 (1) (2000) 65-87.

[27] A. Ishimaru, J. S. Chen, Scattering from very rough surfaces based on the modified second-order Kirchhoff approximation with angular and propagation shadowing, The Journal of the Acoustical Society of America 88 (4) (1990) $1877-1883$. 